\title{
BOLOMETRIC AND UV LIGHT CURVES OF CORE-COLLAPSE SUPERNOVAE
}

\author{
T. A. Pritchard ${ }^{1}$, P. W. A. Roming ${ }^{1,2}$, Peter J. Brown ${ }^{3}$, Amanda J. Bayless ${ }^{2}$, and Lucille H. Frey ${ }^{4,5}$ \\ ${ }^{1}$ Department of Astronomy \& Astrophysics, Penn State University, 525 Davey Lab, University Park, PA 16802, USA \\ 2 Southwest Research Institute, Department of Space Science, 6220 Culebra Road, San Antonio, TX 78238, USA \\ ${ }^{3}$ Department of Physics and Astronomy, George P. and Cynthia Woods Mitchell Institute for Fundamental Physics \& Astronomy, \\ Texas A. \& M. University, 4242 TAMU, College Station, TX 77843, USA \\ ${ }^{4}$ Los Alamos National Laboratory, Los Alamos, NM 87545, USA \\ ${ }^{5}$ Department of Computer Science, University of New Mexico, Albuquerque, NM 87131, USA \\ Received 2013 February 19; accepted 2014 April 11; published 2014 May 14
}

\begin{abstract}
The Swift UV-Optical Telescope (UVOT) has been observing core-collapse supernovae (CCSNe) of all subtypes in the UV and optical since 2005. Here we present 50 CCSNe observed with the Swift UVOT, analyzing their UV properties and behavior. Where we have multiple UV detections in all three UV filters $\left(\lambda_{c}=1928-2600 \AA\right)$, we generate early time bolometric light curves, analyze the properties of these light curves and the UV contribution to them, and derive empirical corrections for the UV-flux contribution to optical-IR based bolometric light curves.
\end{abstract}

Key words: supernovae: general - ultraviolet: general

Online-only material: color figures

\section{INTRODUCTION}

For decades, nearby Type Ia supernovae ( $\mathrm{SNe}$ ) have been extensively studied from the optical to the near-IR (NIR) wavelength range (Filippenko 1997; Krisciunas et al. 2004; Wood-Vasey et al. 2007). UV observations, on the other hand, are historically much more limited and mostly include a handful of bright events from IUE and Hubble Space Telescope (HST; Panagia 2003). More recently, the sample of nearby Type Ia $\mathrm{SNe}$ studied in the UV has dramatically increased (Foley et al. 2008; Brown et al. 2009, 2010; Cooke et al. 2011; Maguire et al. 2012b).

In contrast to Type Ia SNe, core collapse supernovae (CCSNe) have not received the same level of attention. With the emergence of dedicated SN follow-up programs and telescopessuch as the Katzman Automatic Imaging Telescope (Filippenko et al. 2001), Carnegie Supernova Project (Hamuy et al. 2006), Fred Lawrence Whipple Observatory (Matheson et al. 2008), Peters Automated Infrared Imaging Telescope (Bloom et al. 2006), Caltech Core Collapse Program (Gal-Yam et al. 2007), Palomar Transient Factory (Rau et al. 2009; Law et al. 2009), and the efforts of the Center for Astrophysics Supernova (SN) Group - nearby CCSNe are being more frequently monitored in both the optical and NIR wavelength ranges with ground-based telescopes.

Despite this surge of interest, UV studies of nearby CCSNe have lagged behind redder wavelengths, even though the UV is a promising probe of these interesting objects. This lack of UV observations is primarily due to the fact that UV studies blueward of the $U$ band are limited by the availability of space-based UV telescopes. Previous to 2005, 17 CCSNe were observed in the UV, primarily by the IUE and $H S T$ instruments (see Brown et al. 2009 for a complete census of pre-2005 UV observations). Efforts to interpret these observations have underscored the utility of UV observations to better understand CCSNe events. The UV may comprise a substantial portion of the CCSNe bolometric flux, depending on $\mathrm{SNe}$ subtype and epoch, which are important to some numerical models, whereas the early UV cooling behavior is thought to be dependent upon pre-explosion progenitor radius, density distribution, and expansion velocity.
The physics governing a CCSN light curve is the timescale and wavelength dependence of the diffusion of photons as radiation is transported toward the surface to escape (Hoeflich et al. 1996). The resultant light curves for stripped envelope CCSNe (Type $\mathrm{Ib} / \mathrm{c}$ and $\mathrm{IIb}$ ) are principally due to the radioactive decay of ${ }^{56} \mathrm{Ni} \rightarrow{ }^{56} \mathrm{Co} \rightarrow{ }^{56} \mathrm{Fe}$ (Tominaga et al. 2005). Observationally, we can break CCSNe down into several subtypes depending on observed light curve and spectral characteristics (Filippenko 2005), which are thought to have progenitor main sequence stars primarily differentiated by mass (Smartt 2009). Type II SNe, that is, $\mathrm{SNe}$ with hydrogen in their spectra, are broken down into IIn $\mathrm{SNe}$, which exhibit narrow hydrogen emission lines; IIP, which have a long lived ( $\sim 100$ day) optical plateau; IIL, which have a linear light curve decline after peak brightness; and IIb, which show hydrogen soon after explosion and then rapidly evolve with weakening $\mathrm{H}$ lines and the development of $\mathrm{He}$ lines. Type $\mathrm{Ib} / \mathrm{c}$ $\mathrm{SNe}$ are relatively similar in that their spectra show no hydrogen, but may or may not show helium lines for $\mathrm{Ib} / \mathrm{c}$, respectively. From a physical standpoint these differences are all thought to be related to the mass of the progenitor and the amount of hydrogen envelope remaining upon explosion. For hydrogen-rich envelope CCSNe (i.e., Type IIP/L/n) the primary energy source is shock deposited followed by hydrogen recombination in the ejecta. Unlike stripped CCSNe, variations in energy input due to ${ }^{56} \mathrm{Ni}$ mass and its associated radioactive decay in Type IIP $\mathrm{SNe}$ do not significantly affect the light curve shape, but serve instead to modify the plateau lifetime by a few weeks (Kasen \& Woosley 2009). Emergent spectra are dominated by continuum emission with a complex collection of absorption and emission lines bearing evidence of various elements in the optically thin surface region. Recently Dessart \& Hillier (2010), using nonLTE time-dependent radiative-transfer modeling of a CCSNe, found that the evolving UV spectrum is primarily driven by line blanketing and metallicity dependencies. The timing and depth of the iron-peak absorption is thus considered a probe of the amount of these elements near the surface.

Since 2005, the NASA Swift mission (Gehrels et al. 2004) has dramatically improved the number of CCSNe observed in the UV, as well as Type Ia SNe $(\sim 80)$. The Swift satellite has a $30 \mathrm{~cm}$ Ultraviolet/Optical Telescope (UVOT; Roming 
et al. 2005) capable of observing in three UV filters (central wavelengths; $u v w 2: \lambda_{c}=1928 \AA ; u v m 2: \lambda_{c}=2246 \AA$; $u v w 1$ : $\left.\lambda_{c}=2600 \AA\right)$, three optical filters $(u, b, v)$, and a UV and optical grism (Poole et al. 2008). Figure 2 of Poole et al. (2008) provides more information on the filter response curves. The primary mission of the Swift satellite is to detect and monitor gamma ray bursts (GRBs); all SNe science performed with UVOT is secondary to that mission. However, just as $\mathrm{SNe}$ are discovered after explosion via blind searches rather than observations of a known location, the isotropically distributed GRBs must also be detected via blind searches. This isotropic distribution means that Swift can point in the direction of, and observe, any particular SN without affecting the chances of a GRB discovery or the GRB response time. In this sense, UVOT is an ideal UV monitoring instrument with its rapid response to targets of opportunity (ToO) and the ease of submitting observation requests for them. While UVOT may not have the sensitivity or resolution of the HST, these attributes allow UVOT to respond to SNe days faster than HST and obtain more numerous individual observations, making it the workhorse instrument of UV CCSNe studies.

In this paper, we present UV observations of CCSNe as observed by the NASA Swift satellite from launch through early 2012. In Section 2 we discuss the $\mathrm{SNe}$ observations and data reduction pipeline. In Section 3 we examine the properties of the sample light curves and their associated colors. We calculate observed absolute magnitudes, color evolution, and UV decay rate/light curve shape, as well as examine differences in these values based upon SN subtype. In Section 4 we use a particularly well observed subset of this sample to calculate bolometric light curves for these $\mathrm{SNe}$ at early times where the UV flux is a sizable fraction of the total luminosity. We examine these based upon SN subtype, and from these light curves we calculate UV-bolometric corrections based on optical colors for use as an empirical correction to ground-based, optical-IR CCSNe pseudo-bolometric light curves.

\section{OBSERVATIONS}

Swift observations of CCSNe are triggered as ToO observations typically after an $\mathrm{SNe}$ candidate is found via other surveys and reported in the Central Bureau for Electronic Telegrams (CBET), International Astronomical Union Circulars (IAUC), or Astronomer's Telegram (ATEL). Observations of CCSNe are commonly proposed by a number of different science working groups; however, in order to leverage UVOT's UV capabilities most observed SNe have the following characteristics: (1) low line-of-sight galactic reddening $(E(B-V) \lesssim 0.03),(2) \geqslant 10^{\prime \prime}$ separation from the host galactic core to minimize coincidence losses due to a bright background, (3) nearby ( $z \lesssim 0.02$ ), and (4) SNe thought to be discovered "early," such that UV detections are likely. This typically means either a recent pre-explosion upper limit, an observed spectrum with a strong blue continuum, or a best match photometrically or spectrally with a young $\mathrm{CC}$ $\mathrm{SNe}$. These are, of course, guidelines, not search criteria, and have been developed over the course of the mission and are often ignored in the case of uniquely interesting events. This suggests that our sample as presented here is biased, but as the largest sample available, we use it to draw some broad conclusions about the UV behavior of these objects.

Once a ToO has been triggered, Swift usually commences observations using six color filters. A typical observational cadence will vary over the campaign, with observations often starting with a short separation of $\sim 1-2$ days as we examine the early emission and identify UV variability. The cadence then typically lengthens out to $\sim 1$ week as we begin to lose UV detections and a greater integration time is needed. A follow up 6-10 ks observation for the galaxy host-light template subtraction is often observed $\sim 0.5-1$ yr later, if no prior observations of the host galaxy have occurred. A summary of SN observed by Swift and included in this sample may be seen in Table 2. A typical exposure time ranges from $2 \mathrm{ks}$ when the object is bright to 4-6 ks as the SNe fades, and the number of observations vary from $\sim 6-50$. SNe observations without any clear UV detections have been excluded from this paper.

Images were obtained from the NASA HEASARC Swift Archive. All images were processed from the raw image and event files using the recent observations and calibrations, and all photometry measurements performed in this paper were performed using NASA Heasoft v6.12. Aspect corrections were performed manually when the automated processing pipeline failed, and images that were unable to be corrected have been excluded. Swift has an approximately 96 minute orbit, of which a maximum of only $\sim 30$ minutes can be spent observing a single target due to scheduling constraints (such as other observations), telescope pointing constraints (due to the sun, earth, and solar panel illumination), and temperature and momentum constraints. As such, an individual SNe's observations are often spread over multiple orbits, in which case each observation (i.e., segment) was co-added over all orbits to generate a single image. Only limited co-adding was done outside this to keep each observation within a short and well-defined timespan of one day. On occasions when a SN was bright enough to warrant UVOT grism observations, a short UVOT single-filter photometric observation occurred as part of the spectral observation. If a detection occurred in that short snapshot it was reported individually, so there may be multiple observations in the same filter on a different observation ID overlapping in time (if the grism orbits were interspersed with the photometric orbits in the observation schedule).

The Swift UVOT is a photon-counting device and as such there are several differences when compared with a typical optical CCD instrument that must be taken into account when performing photometry upon $\mathrm{SNe}$. The primary concern is coincidence loss of photons due to multiple photons arriving during the detector's readout time (which is similar to pileup as seen in Xray CCDs). Coincidence loss is non-linear above a certain count rate and while the correction for this has been well calibrated for field objects, especially bright point sources, sources on a galaxy background require some special consideration. We follow the basic photometry recipe from Brown et al. (2009) that is designed to account for the particular challenges that SNe present, with some modifications as discussed below to account for an updated instrument calibration and pipeline. We continue to perform aperture photometry using a $3^{\prime \prime}$ aperture. This is smaller than the $5^{\prime \prime}$ aperture recommended by Poole et al. (2008) for use on isolated objects, but due to the fact that this is on the same scale as the UVOT point spread function (PSF), it has been empirically found to lower the contamination to the bright background of the host galaxy (Li et al. 2006). We account for sensitivity variations across the detector via the incorporation of large-scale structure and exposure map, as well as the mission time dependent sensitivity loss, into the uvotsoursce pipeline as discussed in the updated UVOT calibrations in Breeveld et al. (2010). 
Table 1

The Effect of Performing Background Galaxy Template Subtraction on Our Photometry for Those SNe in Our Sample Where We Have Acceptable Images

\begin{tabular}{lccc}
\hline \hline Filter & Mean & $\sigma$ & Maximum \\
\hline$u v w 2$ & 0.11 & 0.25 & 1.35 \\
$u v m 2$ & 0.16 & 0.29 & 1.38 \\
$u v w 1$ & 0.11 & 0.20 & 1.16 \\
$u$ & 0.10 & 0.21 & 1.25 \\
$b$ & 0.08 & 0.19 & 1.15 \\
$v$ & 0.06 & 0.12 & 0.93 \\
\hline
\end{tabular}

Notes. The mean, $\sigma$, and maximum columns represent the mean, standard deviation, and maximum deviation in magnitudes between pre- and posttemplate subtraction photometry among all observations for all supernovae in our sample that have template data available.

A 5" aperture is used to determine the coincidence loss rate so that we remain consistent with the instrumental calibrations, and we add a $3 \%$ uncertainty in quadrature with the Poisson errors in order to estimate the uncertainty due to small scale structure (Brown et al. 2009; Poole et al. 2008). This is a conservative estimate as it is unchanged from previous papers before the advent of the Breeveld et al. (2010) calibrations. Where we have a pre-explosion image or a suitably late observation ( $\gtrsim 6$ months to $1 \mathrm{yr}$ ), we subtract the galaxy count rate from the $\mathrm{SN}+$ galaxy observations. The ability to obtain these observations is constrained by Swift's heavy subscription rate, and therefore of our 49 objects this has been performed for all filters for 27 $\mathrm{SNe}$, in the UV filters only for $3 \mathrm{SNe}$, and not at all for 19 SNe. See Table 2 for the status of an individual SN. The effect of a missing template image varies-when the $\mathrm{SNe}$ are much brighter than the host galaxy the effect is minimal; however, for faint $\mathrm{SNe}$ missing these observations results in the possibility of spurious detections, systematically brighter observations, and a shallower slope than would otherwise be observed. Using our sample observations that have been template subtracted, we compare photometry before and after this process in order to examine the effect that this has upon our data. In Table 1, we show the mean, standard deviation, and maximum difference in magnitudes that the template subtraction process corrects for due to the intrinsic brightness in the host galaxy.

After the extraction of count rates from the $3^{\prime \prime}$ aperture, we use a curve of growth model PSF from Breeveld et al. (2010) to perform aperture corrections to a $5^{\prime \prime}$ aperture for which the instrument photometry is calibrated. We use updated Vega zero points from Breeveld et al. (2011), which also contains Swift AB magnitudes if those are preferred. Individual six color Swift UVOT light curves from our sample $\mathrm{SNe}$, broken up by subtype, may be seen in Figures 1-4 for Types IIn, IIP (divided into two plots by year observed), and $\mathrm{IIb}+\mathrm{Ib} / \mathrm{c}$, respectively. Upper limits and error bars for the individual observations have been omitted for the sake of visibility, however the complete photometry for each object including error bars and upper limits are retrievable at Swift SNe Web site: http://swift.gsfc.nasa.gov/docs/swift/sne/swift_sn.html. The median and maximum error bars respectively for our sample in each swift filter are $u v w 2: 0.14, u v m 2: 0.14, u v w 1: 0.12, u: 0.1$, $b: 0.09, v: 0.08$ and $u v w 2: 0.52, u v m 2: 0.53, u v w 1: 0.52, u: 0.54$, $b: 0.42$, and $v: 0.36$.

\section{UV LIGHT CURVES OF CCSNe}

In Table 2 we present the list of Swift observed CCSNe used in this table. Our sample consists of 49 CCSNe and is inclusive of all major subtypes of CCSNe, including a number of more exotic SNe such as the two GRBe-SN 2006aj and 2010ma (Campana et al. 2006; Starling et al. 2012), the Type IIL SN 2009kr (Elias-Rosa et al. 2010), the Type Ibn SN 2006jc (Pastorello et al. 2007), and several Super Luminous Supernovae (SLSN; 2008am, 2008es, and 2010kd; Gal-Yam 2012). The explosion dates of many of these are uncertain, so we will use the $v$-band peak time and mag for fiducial purposes to shift our UV light as seen in Figure 5. This is the most uniform method available for setting our light curves to comparative timescales, but is suboptimal for the SNe cases where Swift only observes a $v$-band declines. Below, we discuss the observed properties of these SNe broken down by subtype. Dust corrections have not been applied at these wavelengths for Figures 1-7, as the correction is highly dependent upon both the dust model and intrinsic SNe spectrum. However, the Milky Way (MW) line of sight and host $E(B-V)$ have been listed in Table 2 as found in the literature.

\subsection{Type IIn}

The Type IIn SNe in our sample (Figure 5; top panels) show the greatest diversity of UV behavior of all our CCSNe subtype samples. These SNe are often thought to be the product of luminous blue variable (LBV) stars going SNe, where the expanding SNe ejecta interacts with previous LBV mass loss eruptions (often modeled as a wind, or shell ejections) producing the narrow $\mathrm{H} \alpha$ line that characterizes this $\mathrm{SNe}$ subtype (Chatzopoulos et al. 2011; Inserra et al. 2013; Pritchard et al. 2012; Roming et al. 2012; Smith et al. 2012c, 2012b). However, there is some disagreement with this progenitor model and it has also been suggested that these could be related to $\eta \mathrm{Car}$ type outbursts (Humphreys et al. 2012). In the LBV-progenitor model, the observed light curve behavior is produced via a combination of an expanding, cooling hydrogen photosphere driven by the $\mathrm{SNe}$ ejecta and the energy injection interaction with the circumstellar medium (CSM) wind/shells. In terms of the observed light curves, we see a variety of behaviors that may be explained by this physical scenario.

In some IIn SNe, such as SN 2007pk and 2010al, the SN peaks quite early in the several days to a week before Swift observations occur, see Section 3.4, and linearly declines across all UV filters. This is similar to our observations of Type IIP SNe, discussed in Section 3.2. This decay in the light curve appears similar to that seen in much of the IIP sample at early times before the optical filters transition into the plateau phase, with an average decay rate of $\sim 0.27 \mathrm{mag} \mathrm{day}^{-1}$ before dropping below Swift detection limits. This is most easily explained by the emission being dominated by the initial SNe ejecta with relatively weak CSM interaction, likely a low-density wind. In a marked contrast, SN 2011ht has a sharp initial rise of $\sim 6$ mag, followed by a gradual rise to maximum and subsequent decay over the next $\sim 100$ days, and finally a very rapid decline of several magnitudes in the UV (and more in the optical) at the final observed upper limit. The differences in behavior of the UV filters are fairly clear in Figure 5, and this behavior is more easily explained by interaction with an optically thick shell. The rapid increase and decrease in brightness would then occur when the obscured shock begins interacting with or finishes passes through the ejecta shell, respectively, and the more gradual rise and fall is moderated by a changing shell and ejecta density/opacity. In between these two cases we observe a variety of intermediate decay rates with a number of the SNe (e.g., 2005ip and 2006jd) demonstrating a 
Table 2

CCSNe Observed by Swift

\begin{tabular}{|c|c|c|c|c|c|c|c|c|c|c|c|c|c|c|c|}
\hline Name & Type & $\begin{array}{l}\text { No. of UV Obs } \\
u v w 2 u v m 2 u v w 1\end{array}$ & $\begin{array}{c}\text { R.A. } \\
\text { (hr m s) }\end{array}$ & $\begin{array}{c}\text { Decl. } \\
(\operatorname{deg~m~s})\end{array}$ & $\begin{array}{c}\text { Galactic } \\
E(B-V)\end{array}$ & Redshift & $\begin{array}{l}\text { Distance } \\
(\mathrm{Mpc})\end{array}$ & $\begin{array}{c}\mu \\
(\mathrm{mag})\end{array}$ & $\begin{array}{c}\text { Host } \\
E(B-V)\end{array}$ & $\begin{array}{l}\text { Upper Limit } \\
2,450,000+\end{array}$ & $\begin{array}{c}\text { Discovery } \\
2,450,000+\end{array}$ & $\begin{array}{c}\text { Shock Breakout } \\
2,450,000+\end{array}$ & $\begin{array}{l}\text { Template } \\
\text { Image }\end{array}$ & $\begin{array}{l}\text { Bolometric } \\
\text { Light Curve }\end{array}$ & Ref: \\
\hline $2005 \mathrm{cs}$ & IIP & $15 / 12 / 12$ & 132952.78 & +47 1035.7 & 0.031 & 0.0015 & $8.9 \pm 0.5$ & $29.75 \pm 0.12$ & 0.01 & & & $3547.6 \pm 0.5$ & $\mathrm{C}$ & $\mathrm{Y}$ & 1,2 \\
\hline $2005 \mathrm{kd}$ & IIn & $7 / 3 / 8$ & 040316.88 & +714318.9 & 0.233 & 0.0150 & $63.3 \pm 4.4$ & $34.01 \pm 0.14$ & 0.15 & & 3683.5 & 3686.8 & I & $\mathrm{N}$ & 3 \\
\hline 2006 $\mathrm{j}^{\mathrm{a}}$ & Ic & $16 / 15 / 16$ & 032139.71 & +165202.6 & 0.126 & 0.0331 & $145.6 \pm 9.7$ & $35.82 \pm 0.14$ & 0.20 & & & 3784.7 & $\mathrm{C}$ & $\mathrm{Y}$ & 4, GRB060218 \\
\hline 2006at & IIP & $11 / 6 / 8$ & 131241.11 & +631645.4 & 0.015 & & & & & & 3802.5 & & $\mathrm{C}$ & $\mathrm{N}$ & 5 \\
\hline $2006 \mathrm{bc}$ & IIP & $4 / 4 / 7$ & 072116.50 & -685957.3 & 0.181 & 0.0045 & $22.7 \pm 4.9$ & $31.73 \pm 0.50$ & 0.33 & 3811.1 & 3819.2 & & $\mathrm{C}$ & $\mathrm{Y}$ & 6,7 \\
\hline 2006bp & IIP & $14 / 13 / 18$ & 115355.74 & +52 2109.4 & 0.026 & 0.0035 & $17.6 \pm 0.8$ & $31.23 \pm 0.13$ & 0.37 & & & $3833.3 \pm 0.4$ & $\mathrm{C}$ & $\mathrm{Y}$ & 8,9 \\
\hline $2006 j \mathrm{c}$ & $\mathrm{Ibn}$ & $15 / 61 / 26$ & 091720.78 & +415432.7 & 0.018 & 0.0056 & $25.8 \pm 2.6$ & $32.06 \pm 0.14$ & 0.03 & 4000.3 & 4018.3 & $4003.0 \pm 50$ & $\mathrm{C}$ & $\mathrm{N}$ & 10,11 \\
\hline 2006jd & IIn & $10 / 11 / 10$ & 080207.43 & +004831.5 & 0.049 & & $83.8 \pm 1.5$ & $34.62 \pm 0.13$ & 0.01 & & 4021.0 & & $\mathrm{C}$ & $\mathrm{Y}$ & 12,13 \\
\hline $2007 Y$ & $\mathrm{Ib}$ & $16 / 4 / 18$ & 030235.92 & -225350.1 & 0.019 & 0.0046 & $18.05 \pm 1.3$ & $31.28 \pm 0.16$ & 0.09 & 4119.7 & 4147.3 & $4145.5 \pm 5.0$ & $\mathrm{C}$ & $\mathrm{Y}$ & 14,15 \\
\hline $2007 a a$ & IIP & 4/3/ 6 & 120027.69 & -010451.6 & 0.023 & 0.0039 & $20.5 \pm 2.6$ & $31.56 \pm 0.14$ & & & 4149.8 & & $\mathrm{C}$ & $\mathrm{N}$ & 16,17 \\
\hline $2007 \mathrm{ck}$ & IIP & $3 / 1 / 7$ & 182305.59 & +295401.0 & 0.097 & 0.0270 & $112.51 \pm 14$ & $35.30 \pm 0.31$ & & & 4178.5 & & $\mathrm{C}$ & $\mathrm{N}$ & 18 \\
\hline 2007od & IIP & $11 / 10 / 9$ & 235548.68 & +18 2454.8 & 0.031 & 0.0058 & $24.50 \pm 1.4$ & $31.91 \pm 0.20$ & 0.09 & 4319.5 & 4406.5 & 4398.5 & C & $\mathrm{Y}$ & $19,20,21$ \\
\hline 2007pk & IIn & $8 / 9 / 10$ & 013147.07 & +333654.1 & 0.046 & 0.0167 & $66.90 \pm 4.7$ & $34.13 \pm 0.15$ & $<0.13$ & 4409.8 & 4414.8 & $4412.2 \pm 2.0$ & $\mathrm{C}$ & $\mathrm{Y}$ & $22,23,24$ \\
\hline 2007uy & $\mathrm{Ib}$ & $1 / 1 / 9$ & 090935.35 & +330708.9 & 0.020 & 0.0065 & $31.0 \pm 2.0$ & $32.46 \pm 0.13$ & & 4452.5 & 4466.1 & & $\mathrm{C}$ & $\mathrm{Y}$ & 25 \\
\hline 2008D & $\mathrm{Ib}$ & $1 / 1 / 1$ & 090930.65 & +330820.3 & 0.020 & 0.0065 & $31.0 \pm 2.0$ & $32.46 \pm 0.13$ & 0.6 & 4474.5 & 4475.1 & 4474.8 & $\mathrm{C}$ & $\mathrm{N}$ & 26 \\
\hline 2008M & IIP & $5 / 5 / 5$ & 062141.28 & -594345.4 & 0.040 & 0.0076 & $40.76 \pm 8.4$ & $33.01 \pm 0.48$ & & 4462.5 & 4483.5 & & $\mathrm{C}$ & $\mathrm{Y}$ & 27 \\
\hline $2008 \mathrm{am}^{\mathrm{b}}$ & IIn & $3 / 2 / 3$ & 122836.25 & +153449.0 & 0.022 & 0.2380 & $950.1 \pm 66$ & $39.89 \pm 0.15$ & & & 4475.4 & 4438.8 & $\mathrm{C}$ & $\mathrm{N}$ & 28,29 \\
\hline 2008aq & IIb & $7 / 7 / 7$ & 125030.42 & -105201.4 & 0.040 & 0.0080 & $31.30 \pm 6.2$ & $32.45 \pm 0.43$ & & 4506.5 & 4523.9 & & $\mathrm{C}$ & $\mathrm{Y}$ & 30 \\
\hline 2008aw & IIP & $6 / 6 / 6$ & 130414.12 & -101912.3 & 0.037 & 0.0104 & $39.12 \pm 5.7$ & $32.94 \pm 0.36$ & & 4507.5 & 4528.0 & & I & $\mathrm{Y}$ & 31 \\
\hline 2008ax & $\mathrm{IIb}$ & $4 / 1 / 7$ & 123040.80 & +413814.5 & 0.019 & 0.0019 & $8.68 \pm 1.2$ & $29.68 \pm 0.31$ & 0.28 & 4528.7 & 4529.0 & 4528.8 & $\mathrm{C}$ & $\mathrm{Y}$ & $32,33,34$ \\
\hline 2008bo & $\mathrm{Ib}$ & $16 / 17 / 28$ & 181954.34 & +743420.9 & 0.053 & 0.0050 & $22.09 \pm 2.5$ & $31.71 \pm 0.25$ & & & 4557.5 & & I & $\mathrm{Y}$ & 35 \\
\hline $2008 \mathrm{es}^{\mathrm{b}}$ & II & $10 / 10 / 9$ & 115649.13 & +542725.0 & 0.010 & 0.2100 & $848.9 \pm 62$ & $39.64 \pm 0.16$ & & & 4582.7 & $4574.5 \pm 1.0$ & I & $\mathrm{Y}$ & $36,37,38$ \\
\hline $2008 \mathrm{ij}$ & IIP & $11 / 9 / 15$ & 181951.81 & +743354.9 & 0.053 & 0.0050 & $22.09 \pm 2.5$ & $31.71 \pm 0.25$ & & 4816.5 & 4819.9 & $4519.2 \pm 2.0$ & $\mathrm{C}$ & $\mathrm{Y}$ & 39 \\
\hline 2008in & IIP & $7 / 6 / 7$ & 122201.77 & +042847.5 & 0.020 & 0.0052 & $13.19 \pm 1.0$ & $30.60 \pm 0.20$ & 0.07 & & 4827.3 & $4825.6 \pm 1.0$ & $\mathrm{C}$ & $\mathrm{Y}$ & 40,41 \\
\hline $2009 \mathrm{~N}$ & IIP & $5 / 5 / 5$ & 123109.47 & -080256.3 & 0.019 & 0.0034 & $12.60 \pm 0.9$ & $30.50 \pm 0.14$ & 0.15 & 4834.5 & 4856.4 & & I & $\mathrm{Y}$ & 42,43 \\
\hline 2009at & IIP & $3 / 3 / 3$ & 134626.68 & +460609.1 & 0.010 & 0.0050 & $24.15 \pm 2.7$ & $31.90 \pm 0.23$ & & 4900.5 & 4902.1 & $4901.3 \pm 1.0$ & $\mathrm{C}$ & $\mathrm{Y}$ & 44 \\
\hline $2009 \mathrm{dd}$ & II & $10 / 10 / 12$ & 120534.10 & +503218.6 & 0.018 & 0.0025 & $16.24 \pm 2.1$ & $31.04 \pm 0.26$ & 0.43 & 4923.5 & 4935.5 & & $\mathrm{C}$ & $\mathrm{Y}$ & $45,46,47$ \\
\hline 2009jf & $\mathrm{Ib}$ & $1 / 13 / 9$ & 230452.98 & +12 1959.5 & 0.100 & 0.0079 & $33.85 \pm 3.0$ & $32.64 \pm 0.20$ & 0.03 & 5097.5 & 5101.8 & $5099.7 \pm 2.0$ & C & $\mathrm{Y}$ & 48,49 \\
\hline $2009 \mathrm{kr}$ & IIL & $22 / 22 / 22$ & 051203.30 & -154152.2 & 0.065 & 0.0065 & $26.16 \pm 5.4$ & $32.03 \pm 0.53$ & 0.01 & 5108.3 & 5142.2 & & I & $\mathrm{Y}$ & 50,51 \\
\hline 2009mg & $\mathrm{IIb}$ & $3 / 1 / 11$ & 062144.86 & -594426.0 & 0.040 & 0.0076 & $40.76 \pm 8.4$ & $33.01 \pm 0.48$ & 0.09 & 5125.5 & 5172.4 & & $\mathrm{C}$ & $\mathrm{Y}$ & 52,53 \\
\hline $2010 \mathrm{~F}$ & IIP & $20 / 20 / 20$ & 100521.05 & -341321.0 & 0.095 & 0.0093 & $32.67 \pm 8.4$ & $32.51 \pm 0.57$ & & 5189.5 & 5209.8 & & I & $\mathrm{Y}$ & 54 \\
\hline 2010ah & Ic & $10 / 3 / 10$ & 114402.99 & +554127.0 & 0.011 & 0.0498 & $208.70 \pm 14$ & $36.60 \pm 0.15$ & & 5246.9 & 5251.0 & & I & $\mathrm{Y}$ & 55,56 \\
\hline 2010al & IIn & $18 / 18 / 18$ & 081415.91 & +182618.2 & 0.016 & 0.0172 & $73.40 \pm 5.1$ & $34.33 \pm 0.15$ & & 5234.5 & 5268.5 & & I & $\mathrm{Y}$ & 57,58 \\
\hline $2010 \mathrm{cr}$ & II & $2 / 1 / 7$ & 132925.04 & +114746.4 & 0.030 & 0.0216 & $97.50 \pm 6.8$ & $34.95 \pm 0.15$ & & 5297.5 & 5302.5 & & I & $\mathrm{Y}$ & 59 \\
\hline $2010 \mathrm{bt}$ & IIn & $8 / 4 / 4$ & 124820.22 & -345716.5 & 0.025 & 0.0162 & $68.70 \pm 4.8$ & $34.19 \pm 0.15$ & & & 5305.6 & & $\mathrm{C}$ & $\mathrm{Y}$ & 61,62 \\
\hline $2010 \mathrm{gs}$ & IIP & $15 / 4 / 13$ & 204539.51 & -053511.0 & 0.048 & 0.0271 & $114.2 \pm 8.0$ & $35.29 \pm 0.15$ & & & 5410.4 & & $\mathrm{U}$ & $\mathrm{Y}$ & 63 \\
\hline 2010j1 & IIn & $24 / 20 / 24$ & 094253.33 & +092941.8 & 0.024 & 0.0107 & $48.80 \pm 3.5$ & $33.44 \pm 0.15$ & 0.06 & 5479.1 & 5503.1 & $5480.0 \pm 5.0$ & $\mathrm{U}$ & $\mathrm{Y}$ & $64,65,66,67$ \\
\hline 2010jp & IIn & 8/9/9 & 061630.63 & -212436.0 & 0.077 & 0.0090 & $38.02 \pm 2.5$ & $32.90 \pm 0.15$ & $<0.16$ & 5491.7 & 5511.8 & & I & $\mathrm{Y}$ & 68,69 \\
\hline $2010 \mathrm{jr}$ & $\mathrm{IIb}$ & $15 / 19 / 28$ & 051934.47 & -323914.6 & 0.015 & 0.0124 & $51.30 \pm 3.6$ & $33.55 \pm 0.15$ & & 5506.8 & 5512.6 & & $\mathrm{C}$ & $\mathrm{Y}$ & 70,71 \\
\hline $2010 \mathrm{kd}^{\mathrm{b}}$ & IIP & 6/6/6 & 120801.11 & +491331.0 & 0.021 & 0.1000 & $414.70 \pm 29$ & $38.09 \pm 0.15$ & & & 5515.0 & & I & $\mathrm{Y}$ & 72 \\
\hline $2010 \mathrm{ma}^{\mathrm{a}}$ & Ic & $7 / 5 / 6$ & 004855.35 & -343359.5 & 0.017 & 0.552 & $2096.5 \pm 100$ & $41.61 \pm 010$ & $<0.03$ & 5549.5 & 5550.0 & & I & $\mathrm{Y}$ & 73, GRB101219B \\
\hline $2011 \mathrm{am}$ & $\mathrm{Ib}$ & $15 / 14 / 15$ & 121626.00 & -431920.0 & 0.117 & 0.0066 & $23.675 \pm 2.2$ & $31.86 \pm 0.21$ & & & 5620.2 & & I & $\mathrm{Y}$ & 74 \\
\hline 2011cj & IIP & $12 / 10 / 11$ & 143253.81 & +113549.3 & 0.024 & 0.0074 & $37.60 \pm 2.6$ & $32.88 \pm 0.15$ & & 5686.9 & 5690.9 & & I & $\mathrm{Y}$ & 75 \\
\hline
\end{tabular}


Table 2

(Continued)

\begin{tabular}{|c|c|c|c|c|c|c|c|c|c|c|c|c|c|c|c|}
\hline Name & Type & $\begin{array}{c}\text { No. of UV Obs } \\
u v w 2 u v m 2 u v w 1\end{array}$ & $\begin{array}{l}\text { R.A. } \\
(\mathrm{hr} \mathrm{m} \mathrm{s})\end{array}$ & $\begin{array}{c}\text { Decl. } \\
(\operatorname{deg} \mathrm{m} \mathrm{s})\end{array}$ & $\begin{array}{c}\text { Galactic } \\
E(B-V)\end{array}$ & Redshift & $\begin{array}{l}\text { Distance } \\
(\mathrm{Mpc})\end{array}$ & $\begin{array}{c}\mu \\
(\mathrm{mag})\end{array}$ & $\begin{array}{c}\text { Host } \\
E(B-V)\end{array}$ & $\begin{array}{l}\text { Upper Limit } \\
2,450,000+\end{array}$ & $\begin{array}{c}\text { Discovery } \\
2,450,000+\end{array}$ & $\begin{array}{c}\text { Shock Breakout } \\
2,450,000+\end{array}$ & $\begin{array}{l}\text { Template } \\
\text { Image }\end{array}$ & $\begin{array}{l}\text { Bolometric } \\
\text { Light Curve }\end{array}$ & Ref: \\
\hline 2011dh & $\mathrm{IIb}$ & $36 / 33 / 34$ & 133005.12 & +471010.8 & 0.031 & 0.0015 & $8.03 \pm 0.7$ & $29.48 \pm 0.25$ & 0.0 & 5712.6 & 5713.4 & $5713.0 \pm 0.4$ & $\mathrm{C}$ & $\mathrm{Y}$ & $76,77,78$ \\
\hline $2011 \mathrm{ht}$ & IIn & $46 / 41 / 48$ & 100810.59 & +515057.0 & 0.009 & 0.0036 & $19.90 \pm 0.2$ & $31.49 \pm 0.25$ & 0.04 & & 5833.7 & & $\mathrm{U}$ & $\mathrm{Y}$ & $79,80,81,82$ \\
\hline $2011 \mathrm{hw}$ & IIn & $5 / 5 / 5$ & 222614.54 & +34 1259.0 & 0.102 & 0.0230 & $96.20 \pm 6.5$ & $34.92 \pm 0.14$ & & & 5874.3 & & I & $\mathrm{N}$ & 84,85 \\
\hline 2011iw & IIn & $4 / 5 / 5$ & 233448.20 & +244501.0 & 0.049 & 0.0230 & $93.40 \pm 6.5$ & $34.85 \pm 0.15$ & & & 5894.6 & & I & $\mathrm{Y}$ & 86 \\
\hline 2012A & IIP & $8 / 4 / 5$ & 102507.39 & +170914.6 & 0.028 & 0.0025 & $8.10 \pm 0.2$ & $29.53 \pm 0.25$ & & 5924.5 & 5933.9 & & I & $\mathrm{Y}$ & 87,88 \\
\hline 2012ak & IIP & $22 / 19 / 22$ & 100127.20 & +364012.0 & 0.016 & 0.0416 & $174.60 \pm 12$ & $36.21 \pm 0.15$ & & & 5979.8 & & I & $\mathrm{Y}$ & 89 \\
\hline 2012aw & IIP & $54 / 40 / 46$ & 104353.76 & +114017.9 & 0.024 & 0.0026 & $10.11 \pm 0.9$ & $30.02 \pm 0.20$ & & 6001.8 & 6003.4 & $6002.6 \pm 0.8$ & $\mathrm{C}$ & $\mathrm{Y}$ & 90,91 \\
\hline 2009 ip $^{c}$ & IIn & $40 / 20 / 23$ & 222308.26 & -285652.4 & 0.017 & 0.0059 & $20.4 \pm 1.6$ & $31.55 \pm 0.21$ & $<0.01$ & & & $6132.5 \pm 1.0$ & I & $\mathrm{Y}$ & $92,93,94,95,96,97$ \\
\hline
\end{tabular}

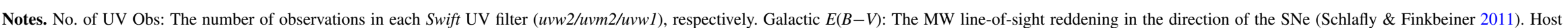

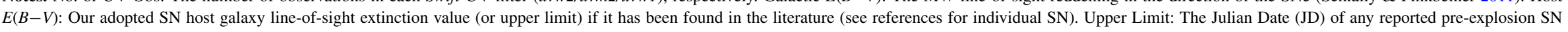

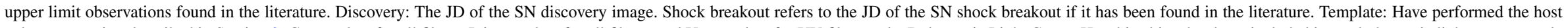

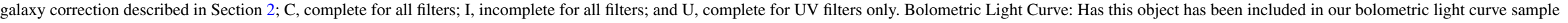
$(\mathrm{Yes} / \mathrm{No})$ ?. References: Literature found on individual SNe that has been used in this table or paper.

${ }^{a}$ Gamma ray burst with visible supernova.

b Super luminous supernova.

c 2012 explosion, not including 2009 and 2010 LBV outbursts.

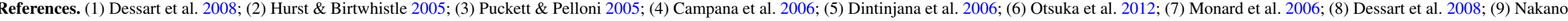

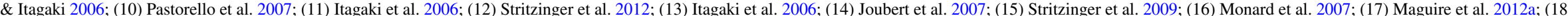

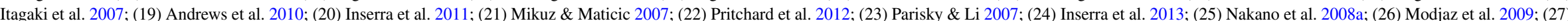

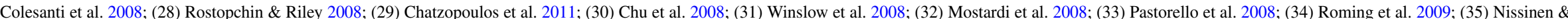

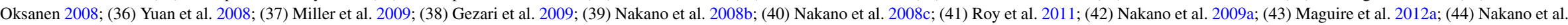

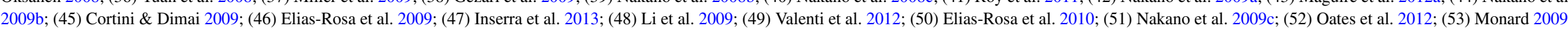

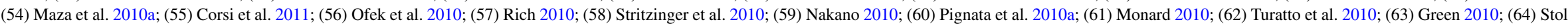

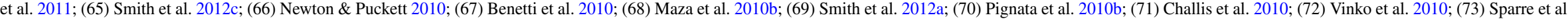

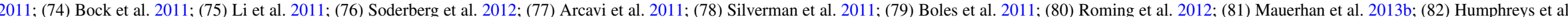

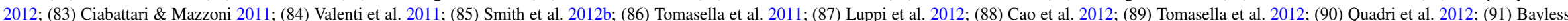
et al. 2013; (92) Drake et al. 2012; (93) Smith \& Mauerhan 2012; (94) Prieto et al. 2013; (95) Levesque et al. 2013; (96) Mauerhan et al. 2013a; (97) Pastorello et al. 2013. 


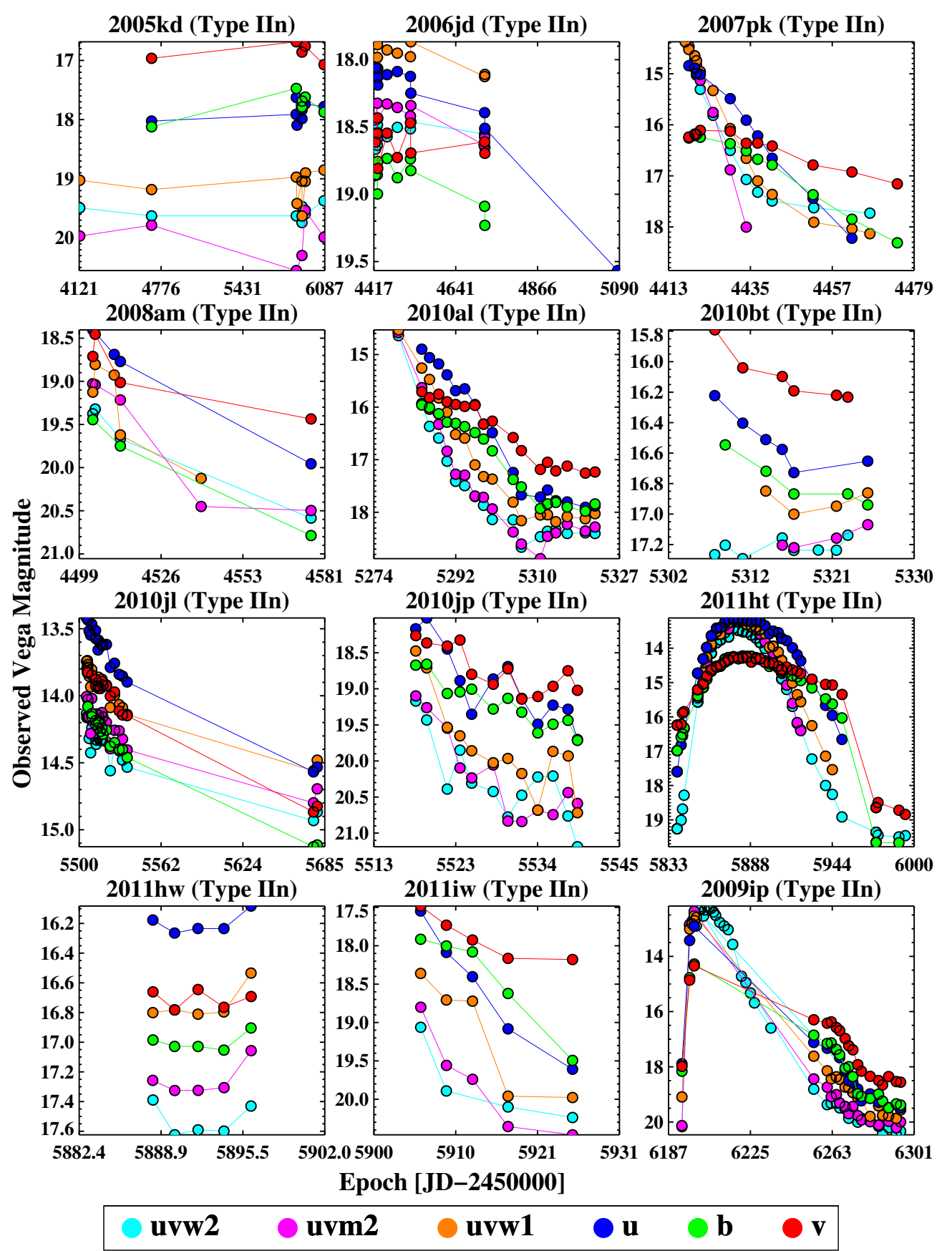

Figure 1. Individual six filter UVOT light curves for the Type IIn SNe in our sample, arranged by date. Observation epochs are the Julian Date (JD 2450000+). (A color version of this figure is available in the online journal.)

long-lived plateau, which we characterize as being driven by energy injection from an optically thin wind or shell. These plateaus have been observed to have UV magnitudes that may be either brighter or fainter than their optical counterparts, and this is primarily dependent upon the CSM density (Smith et al. 2009; Stritzinger et al. 2012)

\subsection{Type $I I / I I P$}

The Type IIP SNe in our sample are our most homogeneous subtype. This tracks with our expectation from the optical light curves as well, because this subtype is characterized by $\sim 100$ day optical plateaus whose brightness and duration behave homogeneously throughout the subtype (compared to observed behavior inside other CCSNe subtypes) and whose variations are thought to be correlated with observables such that they may serve as standard candles (Hamuy \& Pinto 2002; Dessart et al. 2008; Kasen \& Woosley 2009). These SNe are thought to have a thick hydrogen envelope that, when ejected, is optically thick and roughly symmetric. The plateaus are thought to be caused by a combination of the diffusion of thermal energy from the expanding shockwave into this envelope and a hydrogen recombination wave in the photosphere injecting energy into the ejecta (Chevalier \& Soker 1989; Leonard et al. 2002) after the shock has cooled enough to allow this to occur. This results in the behavior of the photosphere being well modeled by a dilute blackbody whose properties are primarily driven by 


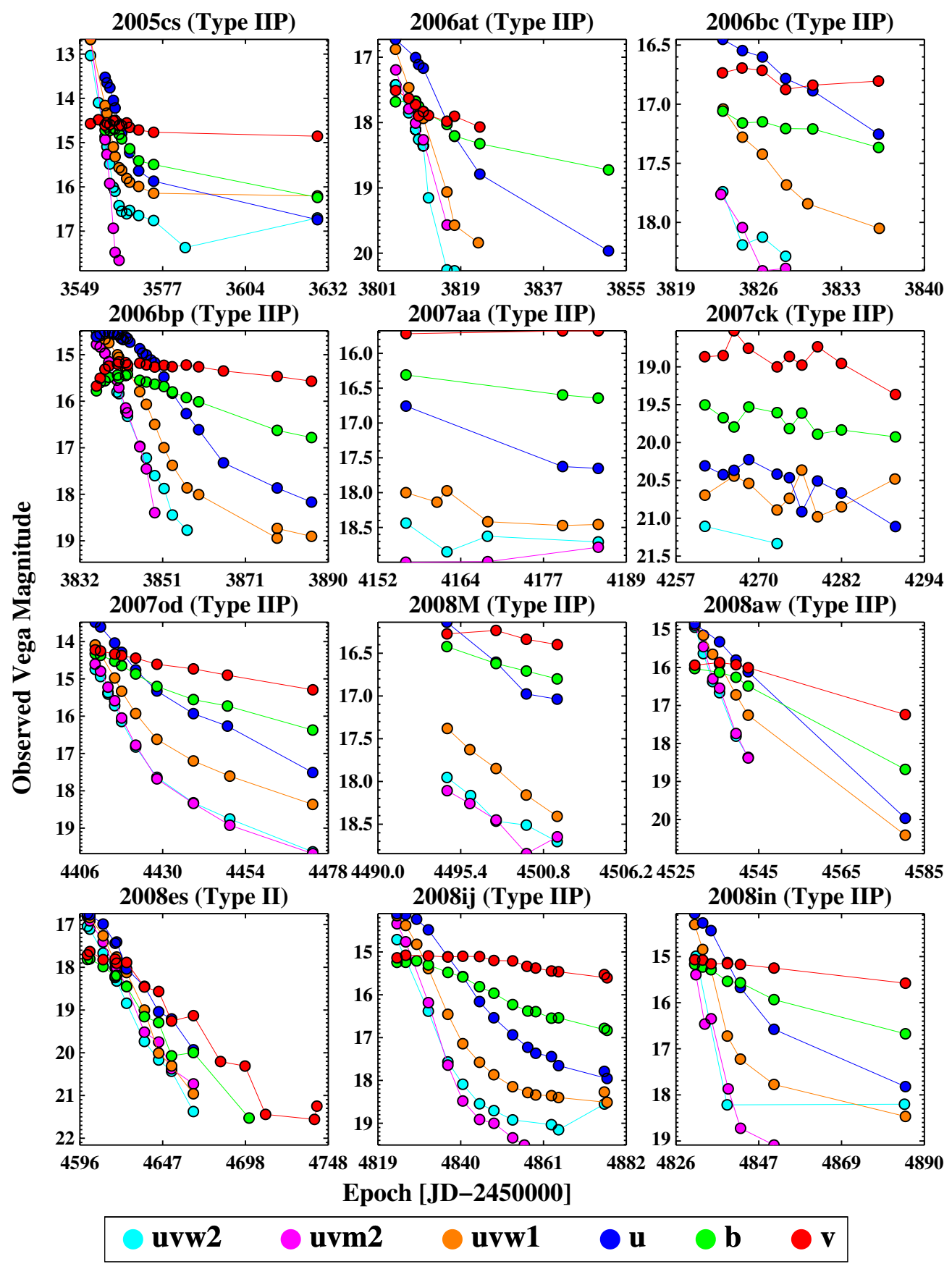

Figure 2. Individual six filter UVOT light curves of the Type II and Type IIP SNe for the years 2005 through 2008 in our sample, arranged by date. Observations are labeled by shortened Julian Date (JD 2,450,000+).

(A color version of this figure is available in the online journal.)

photospheric temperature (Dessart \& Hillier 2005). However, in the UV at temperatures below $\sim 7000 \mathrm{~K}$, iron line blanketing is thought to remove or at least diminish this plateau (Kasen \& Woosley 2009).

The IIP UV (and optical) light curves reach maximum very rapidly - thus it is exceptionally rare to catch any UV rise. Swift observations taken as early as two days after shock breakout do not detect a clear maximum (see Section 3.4 and Figure 6 for more details). As seen in Figure 5, our light curves typically begin $0-10$ days before the $b$-band maximum, where the plateau phase has yet to begin and the light curve declines linearly. This gradually flattens to a plateau portion by 10 days after the $v$-band maximum in those cases where it is detected. This suggests that the hydrogen recombination wave generates a UV plateau in addition to the optical after the photosphere has expanded and cooled from the initially high temperatures of $\sim 15,000-20,000$ $\mathrm{K}$ down to $\sim 5000-7000$; see Dessart et al. (2008), Bersten \& Hamuy (2009), Bayless et al. (2013), and Section 4.4. We do however begin seeing significant deviations from dilute blackbody emission here, which is most likely due to iron line blanketing. This effect is highly temperature and metallicity dependent (Dessart \& Hillier 2010), and will tend to absorb a significant portion of the UV spectrum blueward of 3500 $\AA$ and transform this into optical and IR emission. The large 

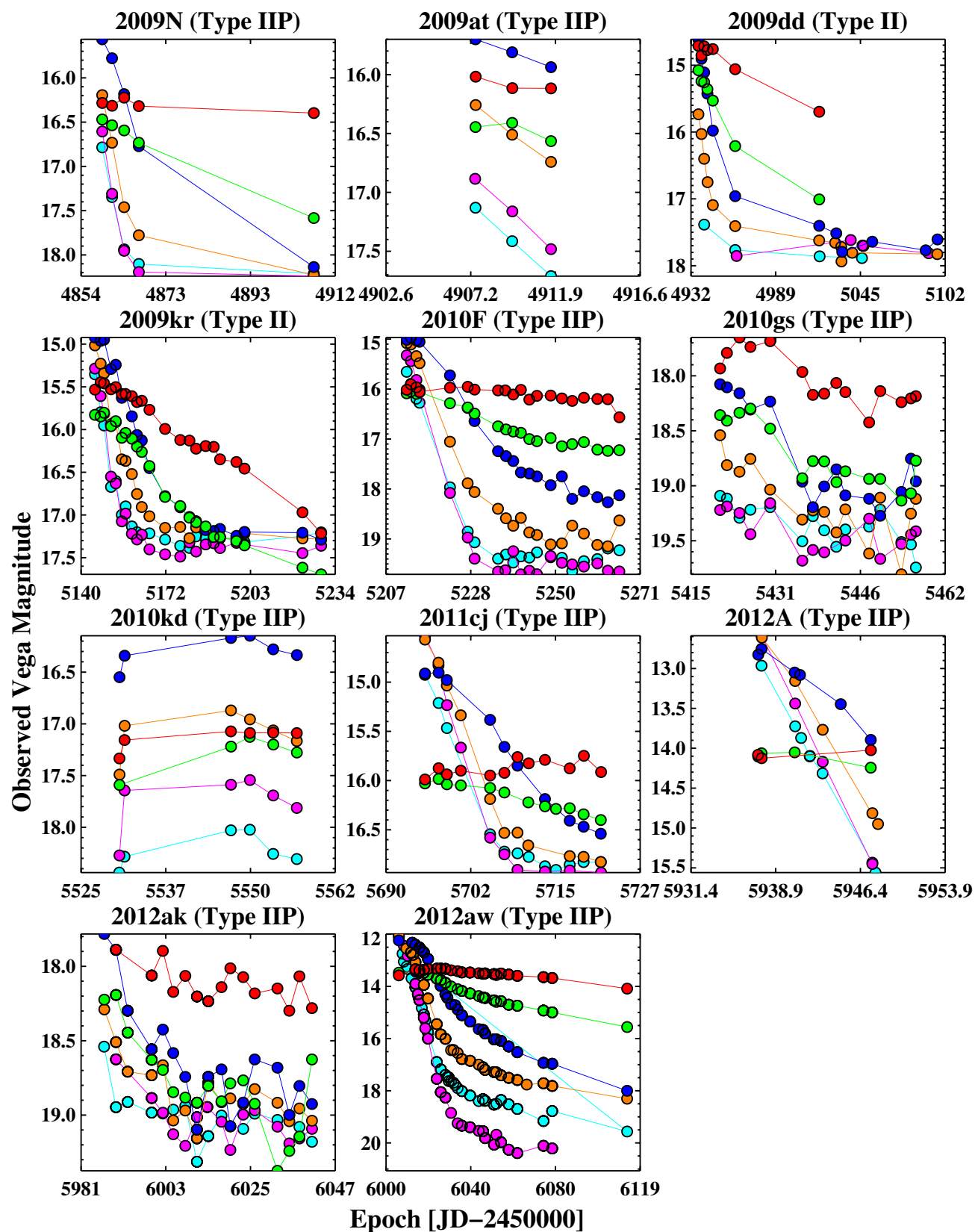

uvw2 uvm2 uvw1

$\mathbf{u} \quad \mathbf{b} \bigcirc \mathbf{v}$

Figure 3. Individual six filter UVOT light curves of the Type II and Type IIP SNe for the years 2009 through 2012 in our sample, arranged by date. Observations are labeled by shortened Julian Date (JD 2,450,000+).

(A color version of this figure is available in the online journal.)

observed spread in the plateau magnitudes would then be due to a combination of intrinsic explosion energy $/{ }^{56} \mathrm{Ni}$ (which has been shown to primarily effect the plateau duration, not brightness (Kasen \& Woosley 2009)), metallically, and dust effects.

\subsection{Type $\mathrm{IIb} / \mathrm{Ib} / \mathrm{c}$}

The "typical" IIb/Ib/c SN, here referred to as stripped CC$\mathrm{SNe}$ (SCCSNe), is UV-faint with relatively few UV detections (Brown et al. 2009; Roming et al. 2009; Oates et al. 2012), owing to its small or nonexistent hydrogen shell. The UV light curves tend to gradually peak and then decline 2-4 mag below the optical filters but otherwise trace the optical behaviors. There are notable exceptions to this rule, however. Sufficiently early observations of the Type IIb SN 2010jr presented here (see Figure 4) have caught the tail of the SN shock breakout cooling phase demonstrating a very early UV bright phase, which may occur in many other SCCSNe if detected early enough. This is caused by the rapid cooling of the SN shock exiting the stellar envelope similar to 2008ax (Roming et al. 2009). We also have two GRB-SNe in this sample. The GRB adds a power-law component to the SNe spectrum that can both distort the light curve shape and cause the SN to be UV-bright at early times. Finally, we have the rather unique Ib/c SLSN that, despite being spectrally similar to the typical $\mathrm{Ib} / \mathrm{cs}$, are thought to have much more massive progenitors and tend to evolve much more slowly. 

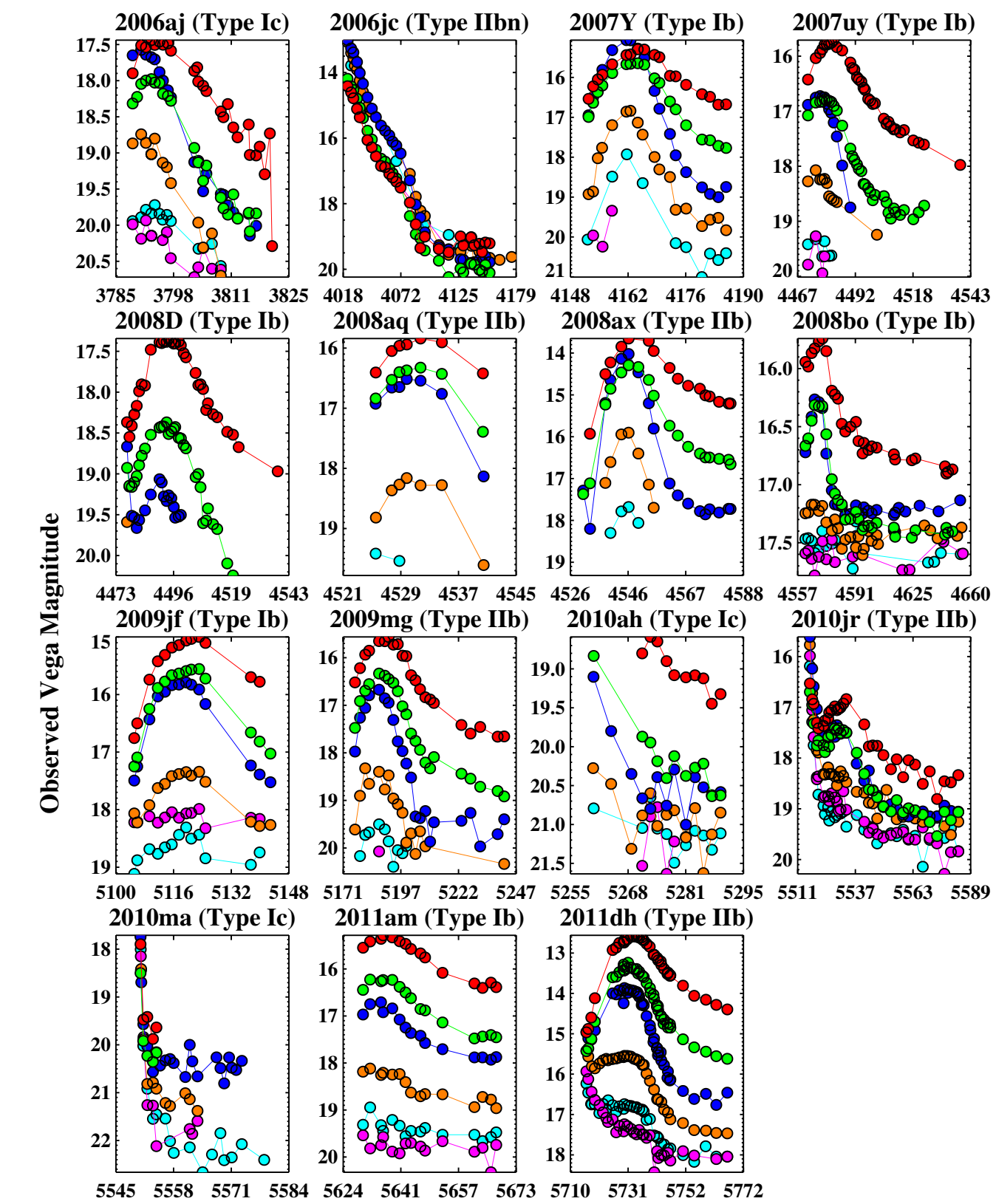

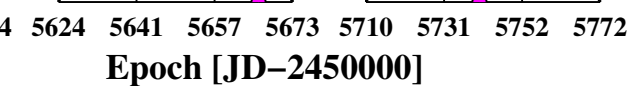

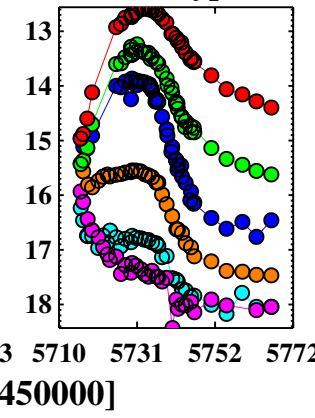

uvw2 uvm2

Figure 4. Individual six filter UVOT light curves of the Stripped CCSNe in our sample, arranged by date. Observations are labeled by shortened Julian Date (JD $2,450,000+)$.

(A color version of this figure is available in the online journal.)

\subsection{UV Rise Time}

An interesting apparent behavior of our sample is that while we are typically observing the maximum brightness in the $v$ band for the SNe shown in Figure 5, we are only seeing UV maximums in a handful of $\mathrm{SNe}$, most of them $\mathrm{SCCSNe}$ - most of our sample IIn and IIPs show no observed maximum, even those that were observed quite early. Early observations of the cooling of the SN shock breakout and initial UV rise are driven by SN shock deposition into the progenitor envelope, and are important for improving models of the initial $\mathrm{SNe}$ explosion. The cooling of the shock breakout and subsequent UV rise happens very rapidly, however, and so is very hard to observe. In particular, Swift SNe observations are dependent upon ground-based survey mission reports of newly discovered $\mathrm{SNe}$ - therefore, even with Swift's rapid response time, which is often much less than one day, the earliest we are able to detect $\mathrm{SNe}$ is still typically several days after the initial shock breakout. In addition to this, the shock breakout date is usually determined by either previous upper limits of the same location by the ground-based survey, or detailed modeling after the fact-both of which are often unavailable. In Figure 6 we present a subset of our larger sample for which we have a relatively well constrained shock breakout date (known to within \pm 3.5 days or less). Using our observations we may then present upper limits on the rise time for this initial UV peak as seen in Table 3. From these it is 


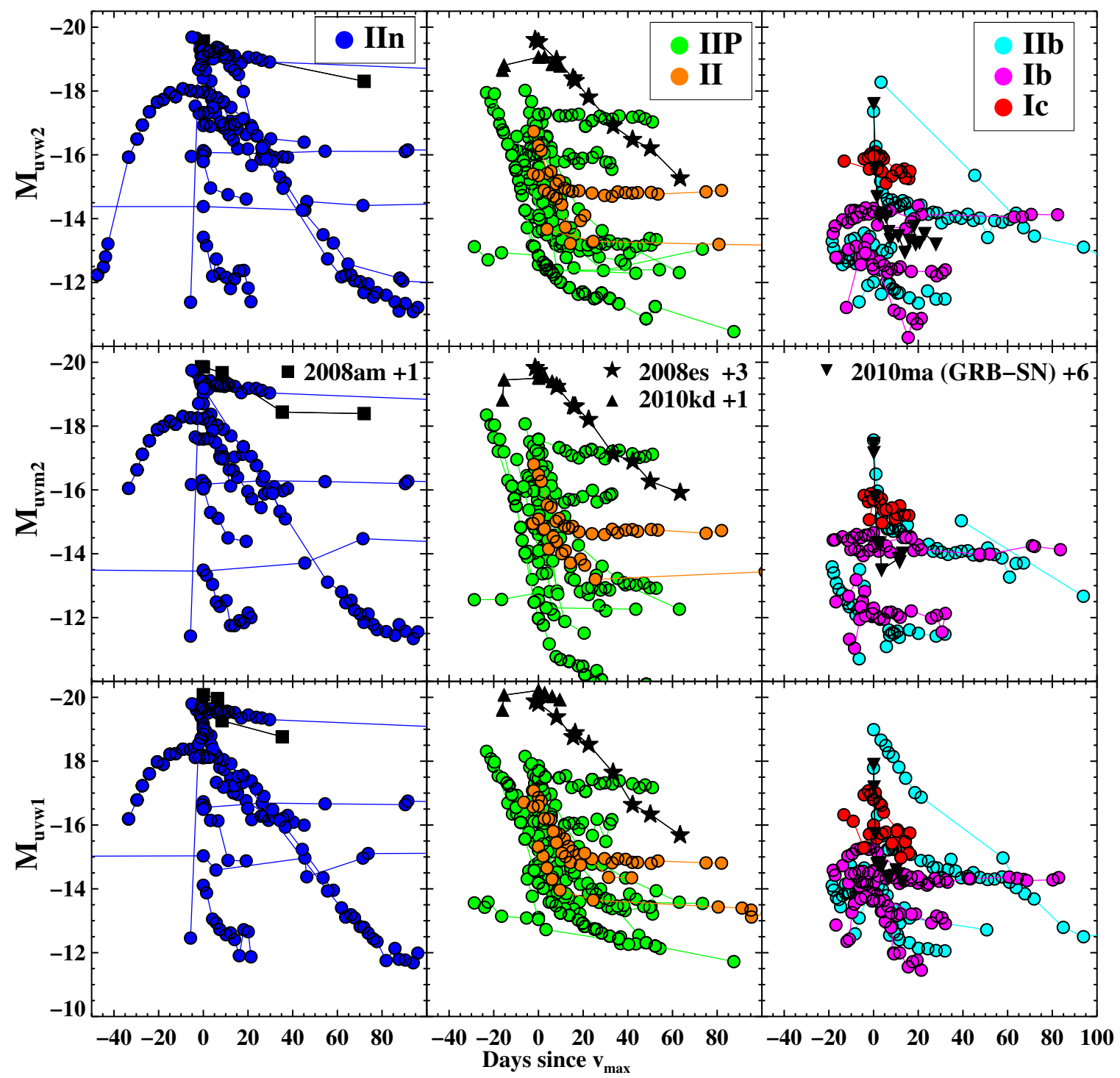

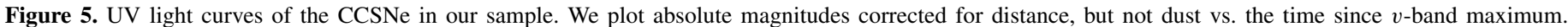

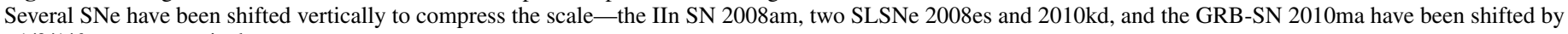
$+1 / 3 / 1 / 6$ mag, respectively.

(A color version of this figure is available in the online journal.)

apparent that this initial rise happens very rapidly across all of our observed subtypes, in less than $\sim 2-5$ days.

\subsection{Absolute Magnitudes}

Using the data from Table 2, we convert our observed magnitudes into absolute magnitudes as seen in Figures 5 and 7. In the optical regime we see that for our sample, our peak observed magnitudes range from $M \sim-18$ to -20 for the IIns, -15 to -18 for the IIPs, and finally -14 to -18 for the IIbcs. In Figure 5 our peak UV magnitudes are fairly similar to that of our optical: the IIns cluster around $M_{u v}=-20$ is brighter than the IIPs in our sample, which are first seen at $M_{\mathrm{Uv}}=-18$ and our stripped CCSNe at $M_{\mathrm{UV}}=-16$. For the IIns and especially the IIPs, peak brightness appears to occur earlier in time, by up to several weeks. There are exceptions to this general trend, which are the more unique $\mathrm{SNe}$ in our sample as detailed previously - the GRB-SNe and SLSN. The absolute magnitudes in the optical colors behave similarly in our sample (Figure 7). In particular, we note the substantial luminosity increase in 2008es, 2010kd, and 2010ma-two SLSNe and a GRB SNe, respectively, which have a clear separation from the rest of the sample.

\subsection{Color Evolution and Comparison with Optical}

In Figures 5 and 6 we noted that there appear to be several general trends for the IIP and SCCSNe in our sample-that is, the IIPs tend to decline rapidly and enter a UV-plateau phase 10-20 days after $v_{\max }$, whereas for the SCCSNe, the UV peaks tend to be around $v_{\max }$ and have a somewhat flatter light curve shape and evolution than the optical. To investigate these trends further, we show the UV $-v$ colors for our SNe plotted against time since $v$-band maximum in Figure 8 . Once again, our IIn sample does not have any clear group behavior if the sample is taken as a whole; however, we do note that there appears to be a number of $\mathrm{SNe}$ with almost flat color evolution between $\mathrm{UV}-v$ colors of 0 and 2 for days -10 to 40 . This behavior is in contrast with the rest of the sample, which indicates a tendency for both rapid increases and decreases in color. 


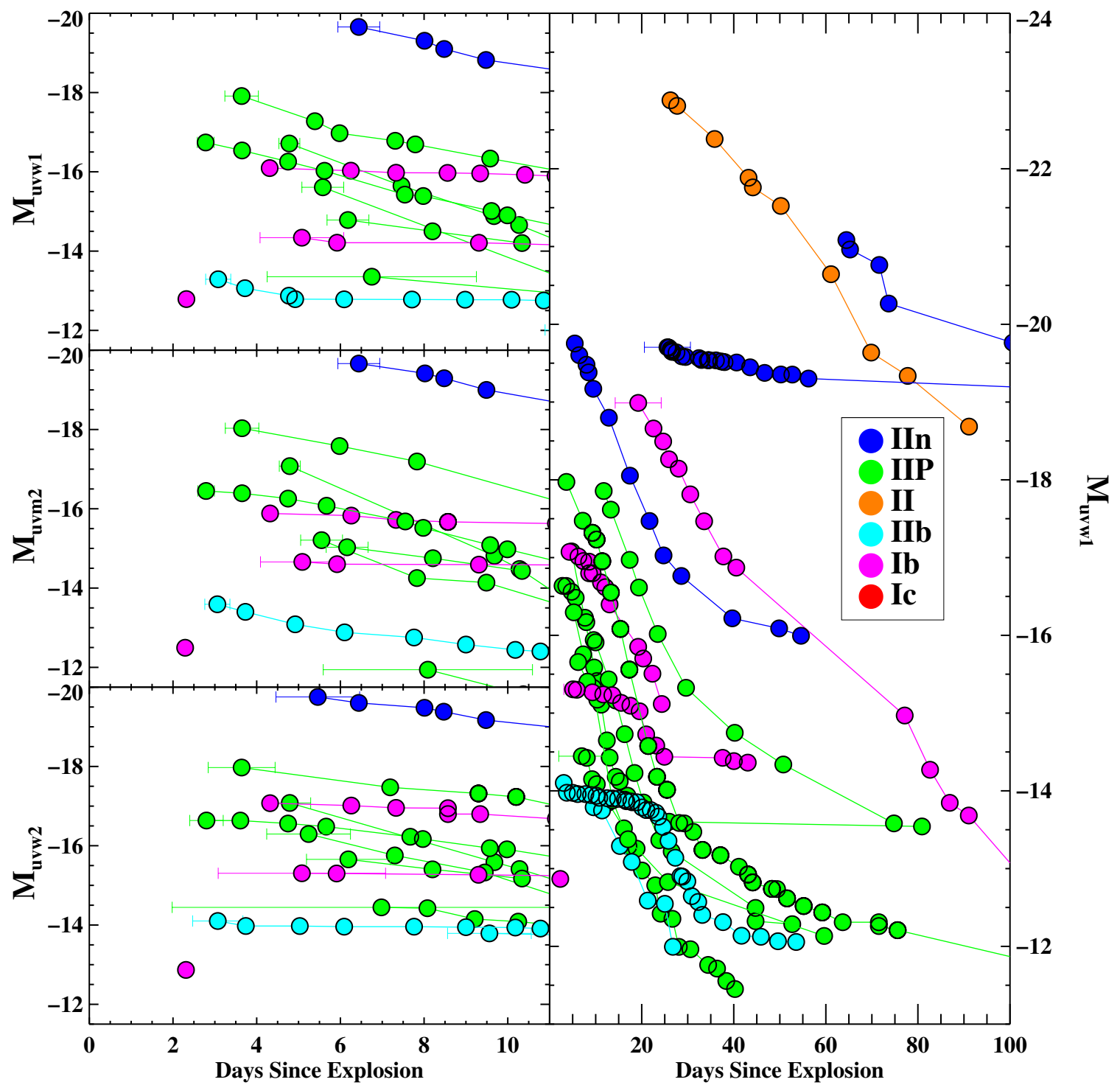

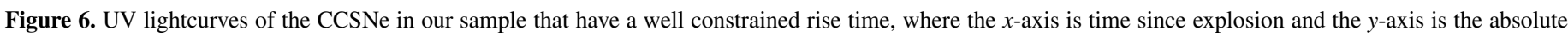

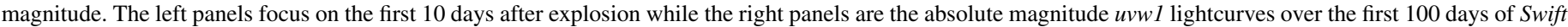
observations.

(A color version of this figure is available in the online journal.)

The IIP SNe however appear to have some common behaviors as you might expect from the more homogenous light curves in Figures 5 and 7 -all of our IIP SNe appear to start very blue at early times, and then plateau at $\sim 10$ days after $v_{\max }$, corresponding to when both the optical $v$-band light curve and the UV-light curves are in the plateau phase. Once again the 1-4 mag spread in plateau color should have a lower intrinsic color that is enhanced by differential reddening in the sample, which has not been corrected for here, as precise extinction corrections in the UV tend to have large errors due to the significant effect that variations in the $2175 \AA$ bump cause.

The variety of SCCSNe that Swift has observed also have some homogenous characteristics. If we reference Figure 7, we note that most of the optical light curves in this sample follow the canonical behavior of a $\sim 20$ day rise with all bands peaking at near the same time, followed by a further $\sim 20$ day decay that transforms into a slower decline at days 30-60 as radioactive heating becomes the primary energy source for the $\mathrm{SNe}$. The UV brightness in these objects is almost always several magnitudes fainter than the optical. While the UV maximum traces the optical maximum, the overall peak is shallower and less pronounced. This faint, shallow UV peak compared to the bright, more pronounced optical peak leads to the evident curvature visible in the UV $-v$ colors.

\section{BOLOMETRIC LIGHT CURVES}

At early times, a sizable fraction of a CCSNe's bolometric luminosity is in the UV bands. Using a well observed subsample, which contains multiple observations in all UV and optical filters as identified in column 12 of Table 2, we generate bolometric light curves as seen in Figure 9. We examine the UV characteristics of and contribution to the bolometric light curves as a function of subtype, and derive empirically based UV corrections for optical bolometric light curves. We calculate these light curves in the following manner. Using the Schlafly $\&$ Finkbeiner (2011) extinction value for the galactic lineof-sight extinction component, we generate a range of model blackbodies at different temperatures that have been redshifted 


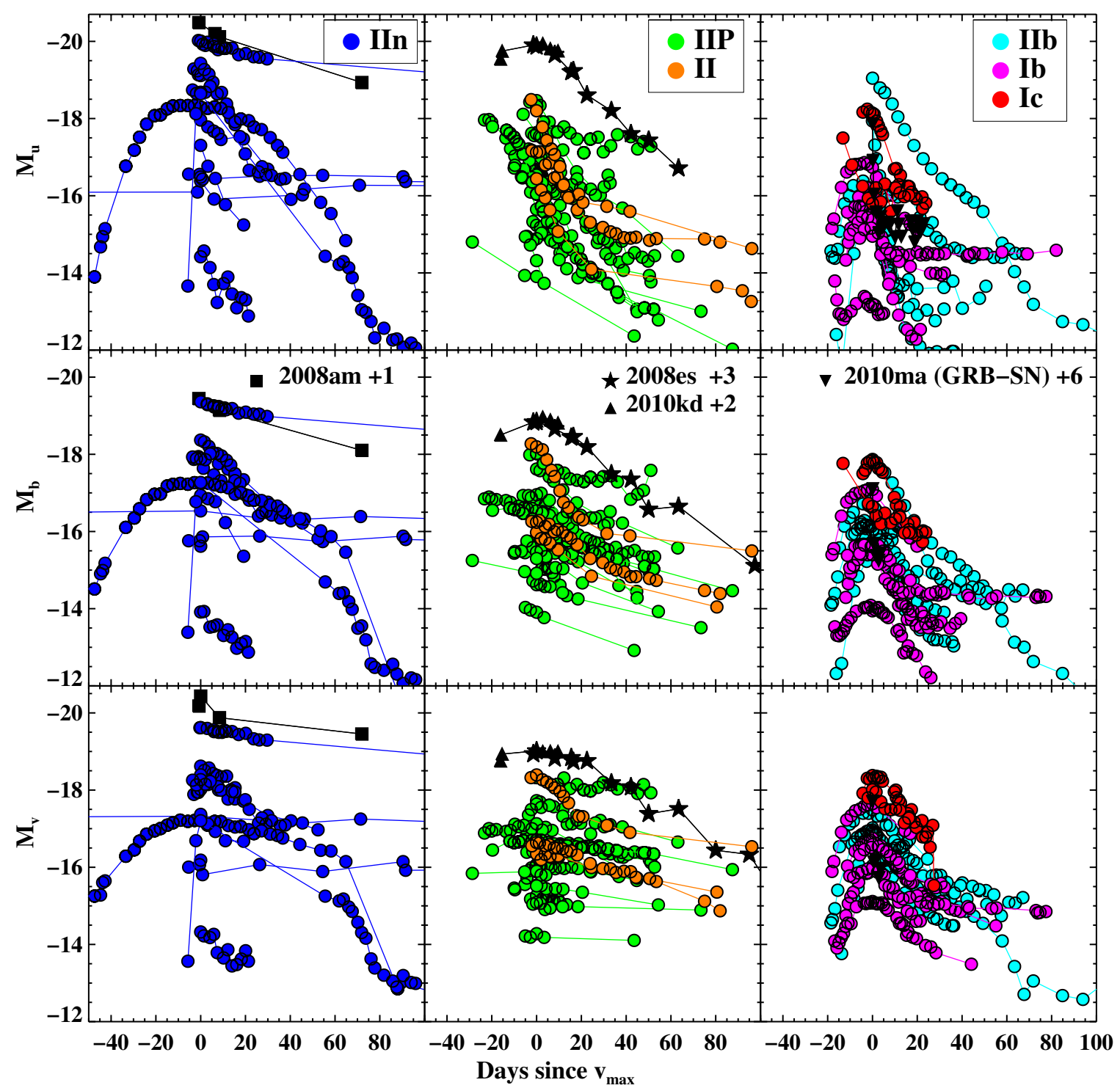

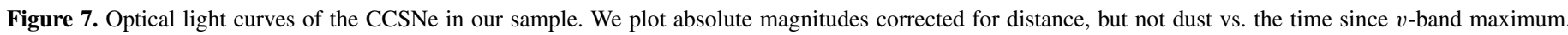

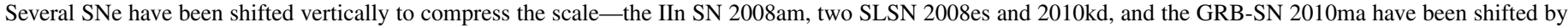
$+1 / 3 / 2 / 6$ mag, respectively.

(A color version of this figure is available in the online journal.)

to the appropriate value and have galactic extinction applied using the Cardelli et al. (1989) analytic model. If the host extinction for the $\mathrm{SNe}$ has been determined in the literature, we apply another Cardelli et al. (1989) model with this value as well. Otherwise, we fit for this host value using an upper limit available in the literature, if possible, or with an upper limit of $E(B-V)=0.3$ if none has been published. We then perform synthetic photometry upon these model spectral energy distributions (SEDs) and minimize the $\chi^{2}$ fit parameter to determine a best-fit model black body temperature and host galaxy reddening where appropriate. At cooler temperatures much of the SNe flux is redward of Swift UV observations, while line blanketing starts causing the UV filters to deviate significantly from the blackbody approximation. This UV deficit caused by line blanketing is degenerate with the $2175 \AA$ bump and our fits are of lower quality (see Section 4.1 for further discussion). We therefore institute a temperature cut of 10,000 $\mathrm{K}$ below which we do not use our fits to calculate the bestfit extinction. What epoch this temperature corresponds to in practice is highly variable upon subtype. For the stripped CCSNe, typically only the observations around the optical peak (or our rare observations of a cooling tail) have a blackbody temperature $>10,000 \mathrm{~K}$, while for IIP this is $\approx 25-30$ days after shock breakout. As a rather inhomogeneous group, but typically very hot, the IIns vary heavily depending on the light curve, but are often above this cutoff for the majority of Swift observations. Due to the red-leak in the $u v w 2$ and $u v w 1$ filters, the central wavelengths are not always an accurate representation of the average wavelength from which we are observing the flux, but the process of fitting to these blackbody synthetic magnitudes allows us to model the red leak contribution of the observed magnitudes as well as to determine count rate to flux conversion values dynamically from the model spectra, and compare them against interpolated values from Brown et al. (2010). Using these monochromatic flux densities for each filter we then integrate over the filter bandpass using a trapezoidal integration, careful to avoid filter overlap due to the red leak. Since we dynamically calculate count rate to flux conversions to reflect 


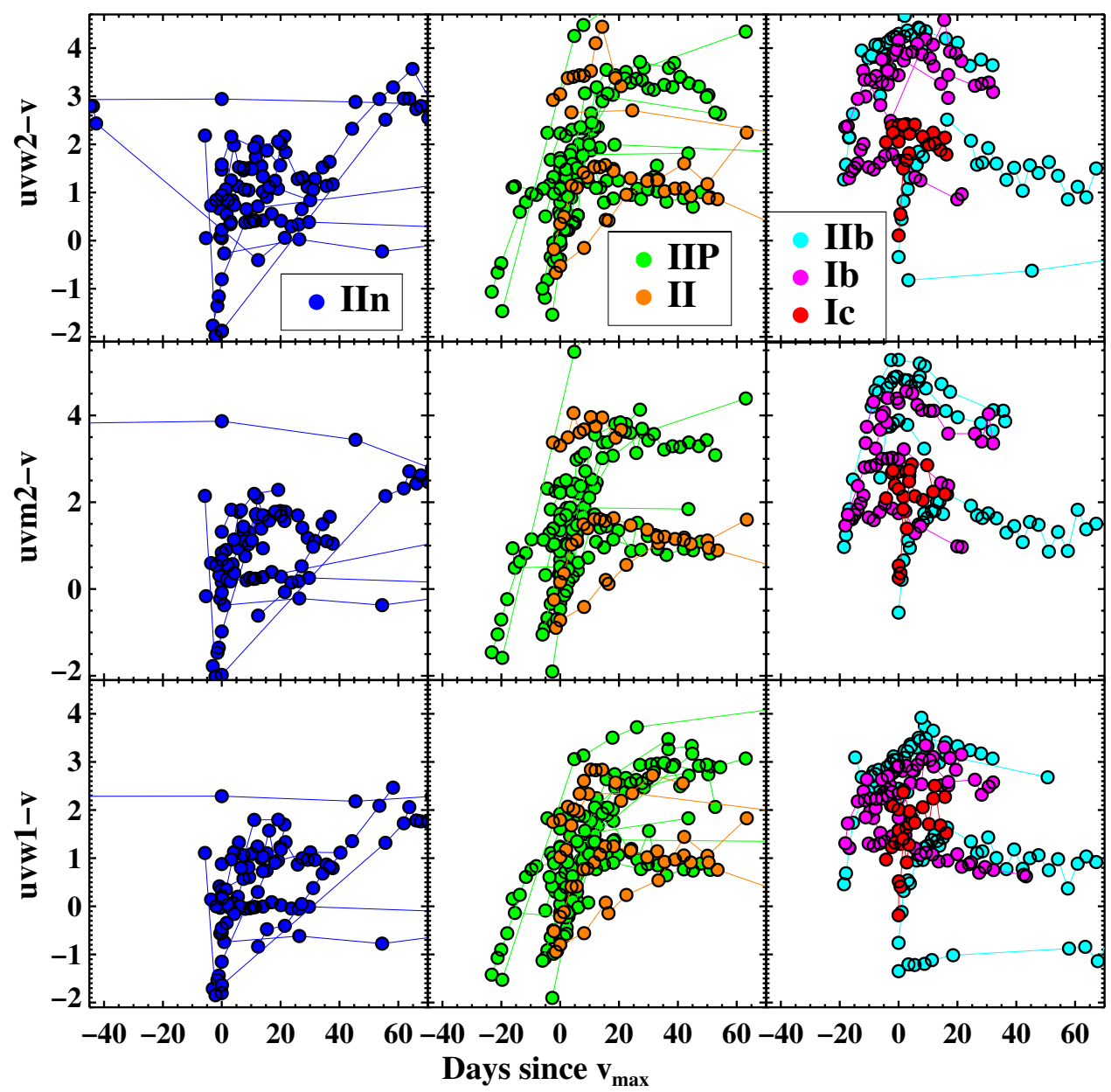

Figure 8. Swift UV $-v$ color light curves. The Type II/IIPs appear to have the most homogenous color curves, followed by the stripped core collapse, and then the Type IIn. We note that the outliers in Figures 5 and 7 are not apparent on these plots and appear to behave as other SNe of their subtype.

(A color version of this figure is available in the online journal.)

Table 3

Days Since Explosion for the Initial Swift Observation

\begin{tabular}{lrrr}
\hline \hline Name & $u v w 2$ & $u v m 2$ & $u v w 1$ \\
\hline $2005 \mathrm{cs}$ & 4.8 & 4.8 & 4.78 \\
$2006 \mathrm{aj}$ & 3.4 & 3.4 & 3.45 \\
$2006 \mathrm{bp}$ & 2.8 & 2.8 & 2.79 \\
$2006 \mathrm{jc}$ & 19.2 & 19.2 & 19.2 \\
$2007 \mathrm{Y}$ & 6.9 & 7.0 & 7.0 \\
$2007 \mathrm{od}$ & 11.7 & 11.7 & 11.7 \\
$2007 \mathrm{pk}$ & 6.4 & 6.4 & 5.5 \\
$2008 \mathrm{D}$ & 2.3 & 2.3 & 2.3 \\
$2008 \mathrm{am}$ & 64.4 & 64.4 & 64.4 \\
$2008 \mathrm{ax}$ & 1.7 & 11.3 & 1.7 \\
$2008 \mathrm{es}$ & 26.2 & 26.2 & 26.2 \\
$2008 \mathrm{ij}$ & 47.5 & 47.5 & 47.5 \\
$2008 \mathrm{in}$ & 5.2 & 5.5 & 5.23 \\
$2009 \mathrm{at}$ & 6.2 & 6.2 & 6.2 \\
$2009 \mathrm{jf}$ & 5.0 & 5.1 & 5.0 \\
$2010 \mathrm{jl}$ & 25.6 & 25.6 & 25.5 \\
$2011 \mathrm{dh}$ & 3.1 & 3.1 & 3.1 \\
$2012 \mathrm{aw}$ & 3.6 & 3.6 & 7.2 \\
\hline
\end{tabular}

our model spectra, and we fit our observed data against model spectrophotometry that includes the UV filter read leak, we are able to eliminate the error in blackbody temperature that typically comes from the filter red leak when calculated via the more typical method of fitting a SED to uncertain filter effective wavelengths in the UV. Instead, we have errors and degeneracies that are driven by deviations of the actual spectra from our model blackbody spectra and the actual reddening versus our applied reddening model. We also compute a bolometric luminosity by applying a far-UV and optical/IR correction to our pseudo-bolometric luminosity (which is the integral of the best-fit blackbody at shorter and longer wavelengths that the UVOT bandpass) for the far-UV and optical+IR corrections, respectively. We use updated UV filter curves from Breeveld et al. (2010), which have a modified red-leak shape from the initial curves depicted in Poole et al. (2008). The SNe 2008ax, 2009mg, 2010cr, and 2011am all have few uvm2 detections but numerous $u v w 2$ and $u v w 1$ detections. In these particular cases we did not use the $u v m 2$ filter in the previously described calculations. Bolometric light curves calculated here are available in machine readable format at the same location as the UVOT photometry files referenced in Section 2.

\subsection{Bolometric Light Curve Flux Completeness and Accuracy}

In using blackbody functions to assist in our handling of the Swift UV-filters red-leaks and calculation of the SNe bolometric light curves, we have introduced some model dependence into these calculations. First, we ask how well we are fitting our results. To examine this we look at the residuals between synthetic magnitudes from our best fit blackbody for our sample 


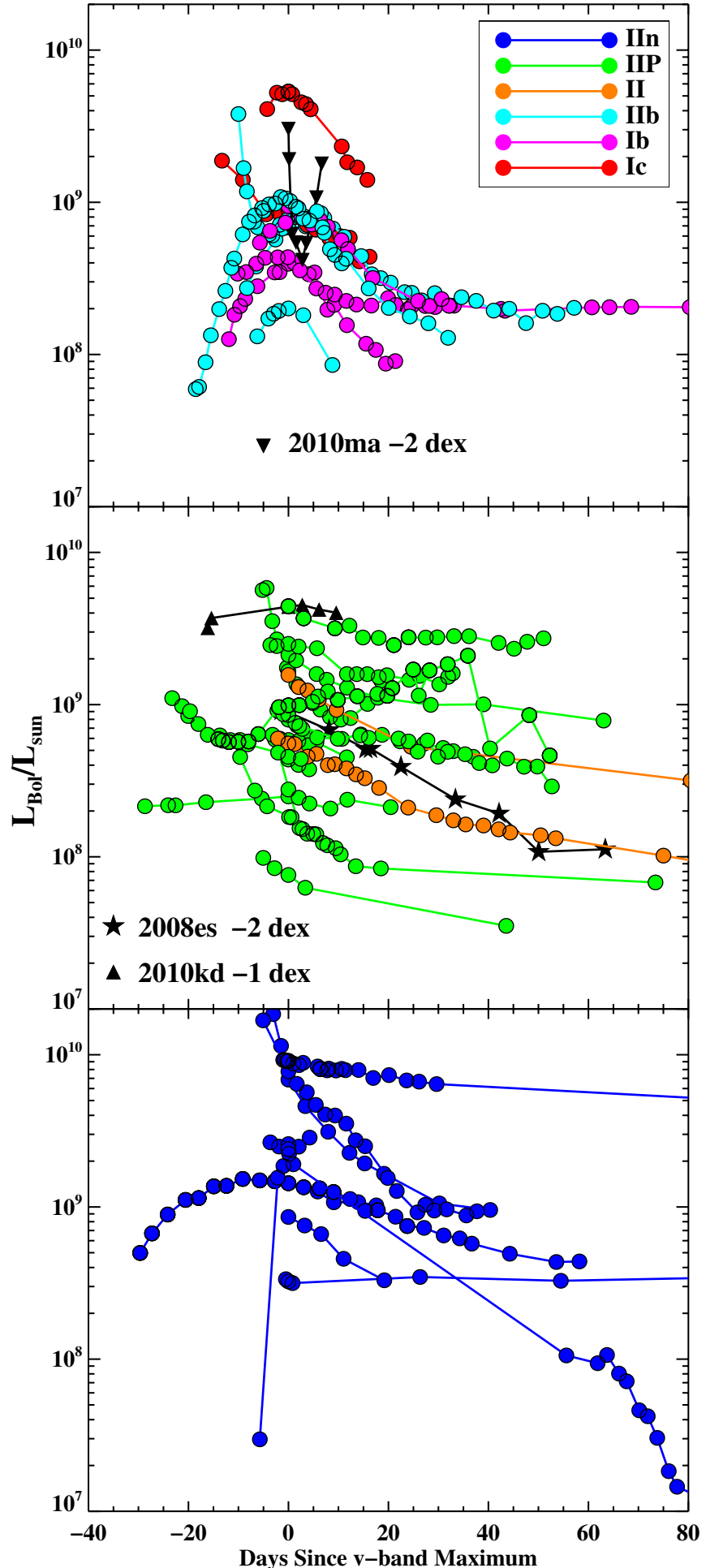

Figure 9. Bolometric light curves from the Swift data arranged by SNe subtypes. The two SLSN 2008es and 2010kd and the GRB-SN 2010ma have had their brightness reduced by a scale factor of 100,10 , and 100, respectively, to bring them in line with the rest of our sample.

(A color version of this figure is available in the online journal.)

in Figure 10 (left). We see that most of our calculations have reasonable residuals compared to our median and maximum photometric errors quoted in Section 2, and at more than $95 \%$ of our epochs our model photometry fits to within the observed $2 \sigma$ photometric errors of the observations, although there are a number of worse fits that suggest that either the observed datapoint is inaccurate (one filter deviating) or that the model is improbable (large errors in several filters). To quantify how accurately these calculations are reproducing observed results, we take a number of $H S T$ UV spectra, combined with ground-based Optical spectra of CCSNe (1993J, 1994I, 1998S, $1999 \mathrm{em}$ ), as well as hydrodynamical models of the Type IIP SNe 2005 cs and 2006bp from Dessart et al. (2008), and generate synthetic magnitudes in the UVOT bands. For 2005cs and 2006bp we used the known values for host reddening listed in Table 2, whereas we fit the other SNe for extinction. We then run these "observations" through our bolometric light curve pipeline and compare our calculated pseudo-bolometric measurement (Swift observed bands only 1600-6000 ̊) with the integrated flux directly from the observed spectra or models. The results are shown in Figure 10. We reproduce these observed values to better than $7 \%$ at temperatures ranging between 5000 and $30000 \mathrm{~K}$. Below $5000 \mathrm{~K}$ this deviation grows as we appear to increasingly underestimate the intrinsic flux as the blackbody peak is redward of the Swift bands. The difference in flux is due to a combination of both error in the fit between the blackbody and the underlying spectral continuum as well as spectral lines/ deviations from a blackbody.

As the primary motivation of these bolometric light curves is to analyze the UV flux contribution to the bolometric luminosity of observed SNe, we must first ask how well we are sampling these SNe with the Swift bandpass. Our observations have a high UV-completeness. In Figure 11 (left) we show our interpolated UV correction for flux that originates blueward of the observed Swift filters as a function of optical color. What we see is that in all but the bluest of observations we are below a $10 \% \mathrm{UV}$ correction factor, and all observations are below a $30 \%$ value. This is a reduction in missed blue flux by a factor of 2.5 to 6 in comparison with what may be done on the ground (cf. Bersten \& Hamuy 2009). Unfortunately, UVOT's reddest filter is the $v$ band, which terminates at $6000 \AA$. This means that we lose a significant portion of the flux as the SNe spectra cool and redden. When the SNe are UV bright, our IR correction may be low at 10\%-20\% (Figure 11, right); however, this increases as the bulk of the flux shifts redward of the optical. In UVOT's worst cases, we sample only 5\%-10\% of the flux for observations of red $\mathrm{SNe}$ (primarily SCCSNe) at late times. We may also combine these two observations to look at the Swift observed fraction of $\mathrm{SNe}$ light as a function of time since the SNe explosion. We find that when we catch these objects early we have a high total flux completeness value as most of the SNe flux is in the UV. By days $\sim 30-40$ the UV brightness has decreased substantially and we are left with primarily optical and IR flux where only $\sim 20 \%$ (or even less in a few rare cases) is in the Swift photometry bands (Figure 12). The Type IIn SNe in our sample appear to deviate from this slightly and have a much longer interaction lifetime due to the CSM interaction with the SNe shock, which helps to keep the light curves UV-bright even at late times. The SLSN also appear to behave on a longer timescales, keeping a high flux completeness at late times. The addition of ground-based red-optical and IR data is necessary to bring these observations up to near flux completeness at later times for most of these SNe. A follow-up paper is in progress where we perform a similar analysis on a subsample of these objects while incorporating comprehensive ground-based observations.

While modeling the spectra of a SNe as a dilute blackbody has long been used as a first order approximation, the presence of metal lines in the UV spectra is expected to deviate from a 

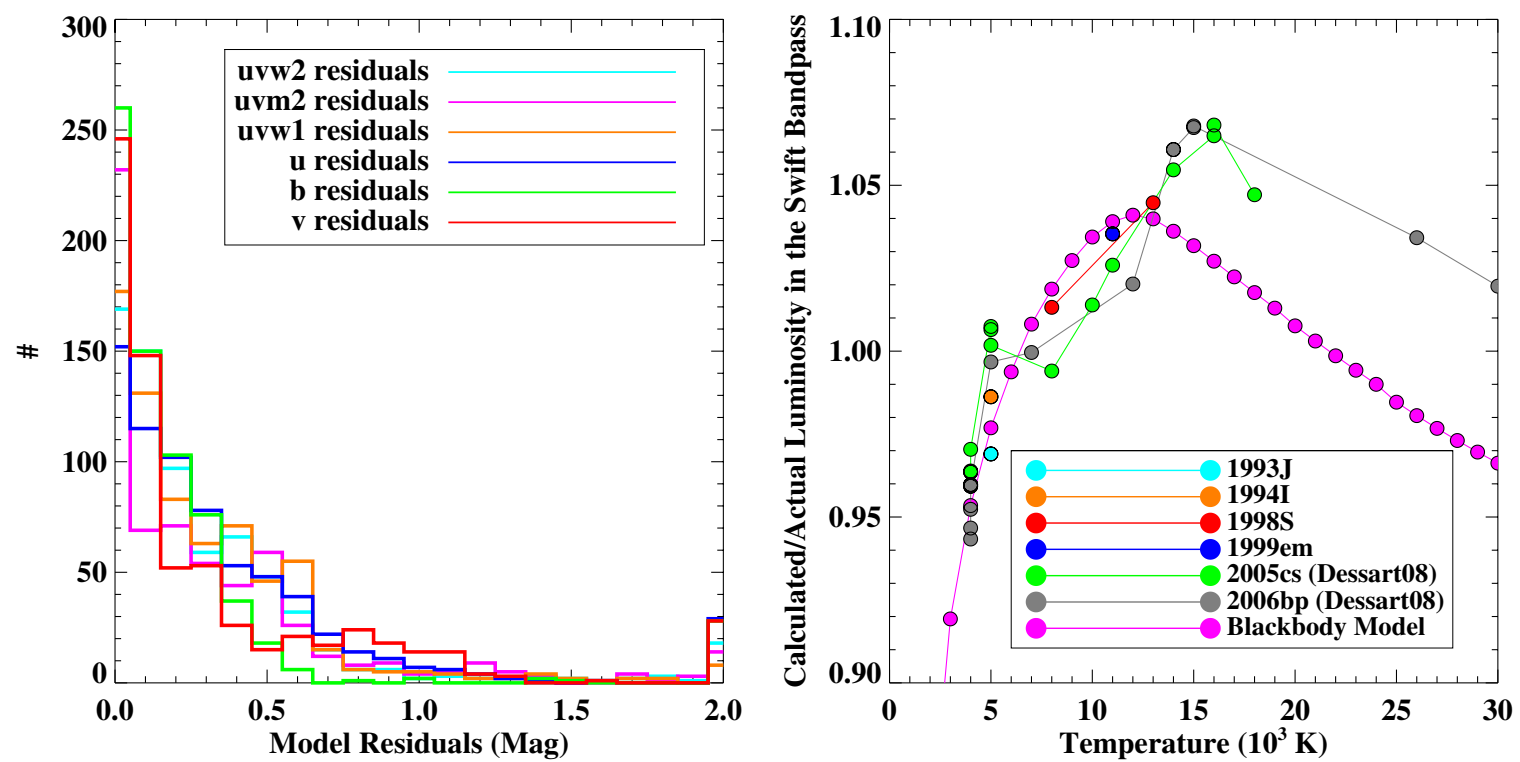

Figure 10. Left: a histogram of our model residuals (i.e., the difference between synthetic magnitudes for our best-fit blackbody model and the observed filter values) per filter for every observation in our sample. Right: a comparison of our calculated peudo-bolometric flux from observed/model spectra and the intrinsic values. (A color version of this figure is available in the online journal.)
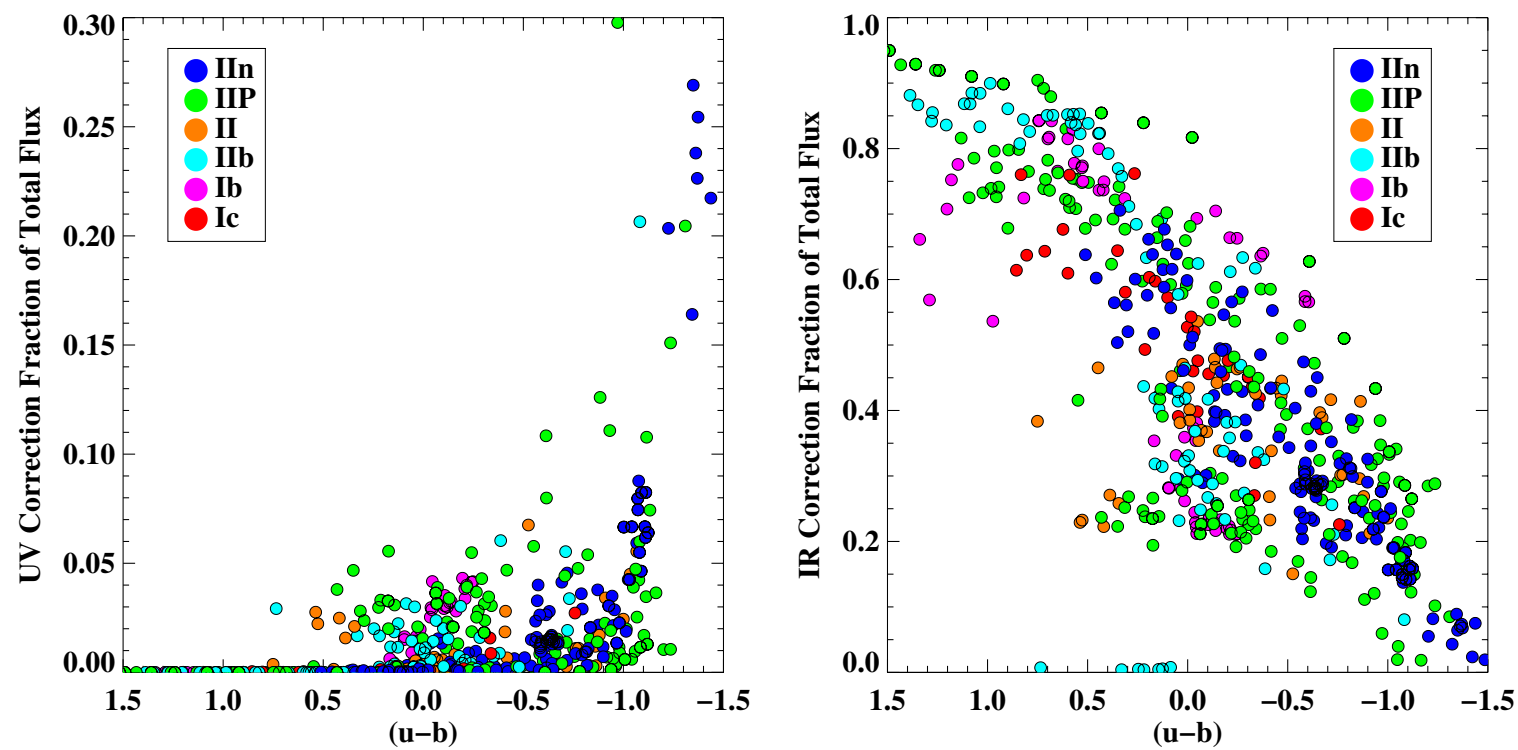

Figure 11. Swift UV (left) and IR (right) correction factors as a function of observed colors.

(A color version of this figure is available in the online journal.)

blackbody. These lines, in addition to residual errors from the extinction correction and the uncertainty in fit at some epochs due to the limited flux in the Swift bandpass are expected to generate some appreciable error. To quantify this, we use hydrodynamic models of the two Type IIP SNe 2005cs and 2006bp as presented in Dessart et al. (2008). Using these UV-optical model spectra, which have well-defined photospheric temperatures, we generate synthetic Swift magnitudes and run these through our fitting algorithms to examine how our measurements compare to the model parameters. We find that at temperatures that are hotter than about $9,000 \mathrm{~K}$, our measured temperatures are systematically biased and about $20 \%$ cooler than the model's photospheric temperature, while below $9000 \mathrm{~K}$ the photospheric temperature tends to be $\sim 40 \%$ hotter. For temperatures above $8000 \mathrm{~K}$ we attribute this bias primarily to the depressed model flux, compared to the blackbody values lowering the best-fit temperatures. At the lower temperatures our flux-completeness becomes rather low as little of the flux is in the UV and the primary bias there is due to the high uncertainties in the UV observations and fitting. This may be seen in Figure 13 (right). Since our IR correction is driven by the fit temperature, the deviation from the model blackbody suggests that at the coolest observations, our worst case, we are underestimating the flux in the IR tail by a factor of $\sim 2$ if we assume a perfect black body, while above this we are often overestimating it where it is both much smaller and a smaller fraction of the total flux.

\subsection{Bolometric and UV Corrections}

For SNe that lack IR and UV observations, it can be convenient to define a bolometric correction value, that is, a value that transforms an observed optical $V$-band value into a 


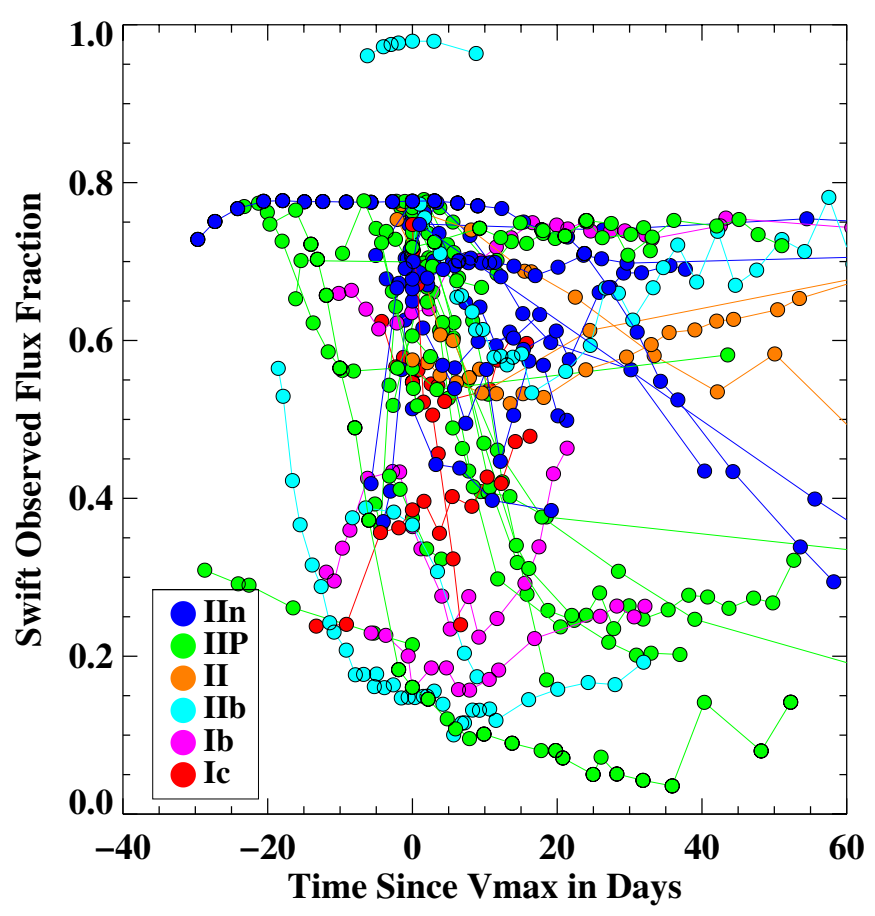

Figure 12. Observed fraction of the $\mathrm{SNe}$ bolometric flux vs. time since $v$-band maximum. As the CSM interaction drives the bolometric luminosity, the IIns in our sample exhibit a large UV flux at much later times than the other CCSNe in our sample. Indeed, for most other SNe in the UVOT observed flux decreases to about $20 \%$ some time between days 20 and 40, while IIns remain more variable and UV bright at later times.

(A color version of this figure is available in the online journal.)

bolometric magnitude empirically using a different observed sample. While we lack IR observations in this data set, many of our observations are early enough that this is not a significant handicap. From our sample fits, $\sim 50 \%$ of our observations have an IR correction of less than $30 \%$, and $\sim 70 \%$ of our observations have an IR correction of less than $50 \%$. We are then able to calculate this conversion as

$$
\mathrm{BC}=m_{\mathrm{bol}}-\left[V-A_{V}\right]
$$

where $\mathrm{BC}$ is the bolometric correction, $m_{\text {bol }}$ is the total bolometric magnitude, $V$ is the observed $v$-band magnitude, and $A_{V}$ is the visual extinction. Bolometric corrections for the $\mathrm{SNe}$ in this sample may be seen in Figure 14. We calculate polynomial fits to this data, which are listed in Equations (2) and (3):

$$
\begin{gathered}
\mathrm{BC}(u-b)=-0.6133-0.517 \times(u-b)-0.4326 \times(u-b)^{2}, \\
\mathrm{BC}(b-v)=-0.4888-1.5046 \times(b-v)-0.9697 \\
\times(b-v)^{2}-0.6768 \times(b-v)^{3} .
\end{gathered}
$$

While this is useful, the proliferation of ground-based IR transient telescopes means that rather than focusing on a total bolometric correction, we should perhaps leverage Swift's unique strengths and instead give a total UV correction, where we supply a magnitude correction for the SNe flux blueward of the $b$ band. This is magnitude value calculated similarly to the bolometric correction (Equation (1) discussed above, but $m_{\text {bol }}$ is instead $m_{u v}$ ) and as such is a distance independent value. In Figure 15 we plot this value versus $u-b$ colors (left) and $b-v$ colors (right).

We include the $u-b$ colors for use with Swift UVOT observations as an estimate for when UV filters might be lacking. Care should be taken not to use these with the more common Johnson U filter, as the Swift filter has a cutoff blueward of the Johnson $U$ band and thus cannot be applied to ground based data. This does illustrate the point that the space $u$ band is a much more efficient tracer of the UV flux than the other Swift optical filters, since it is both closer in wavelength and similarly effected by spectral effects such as line blanketing. We perform a linear fit for $u-b$ and $b-v$, respectively, with the best-fit values listen in Equations (4) and (5), respectively. The standard deviation of
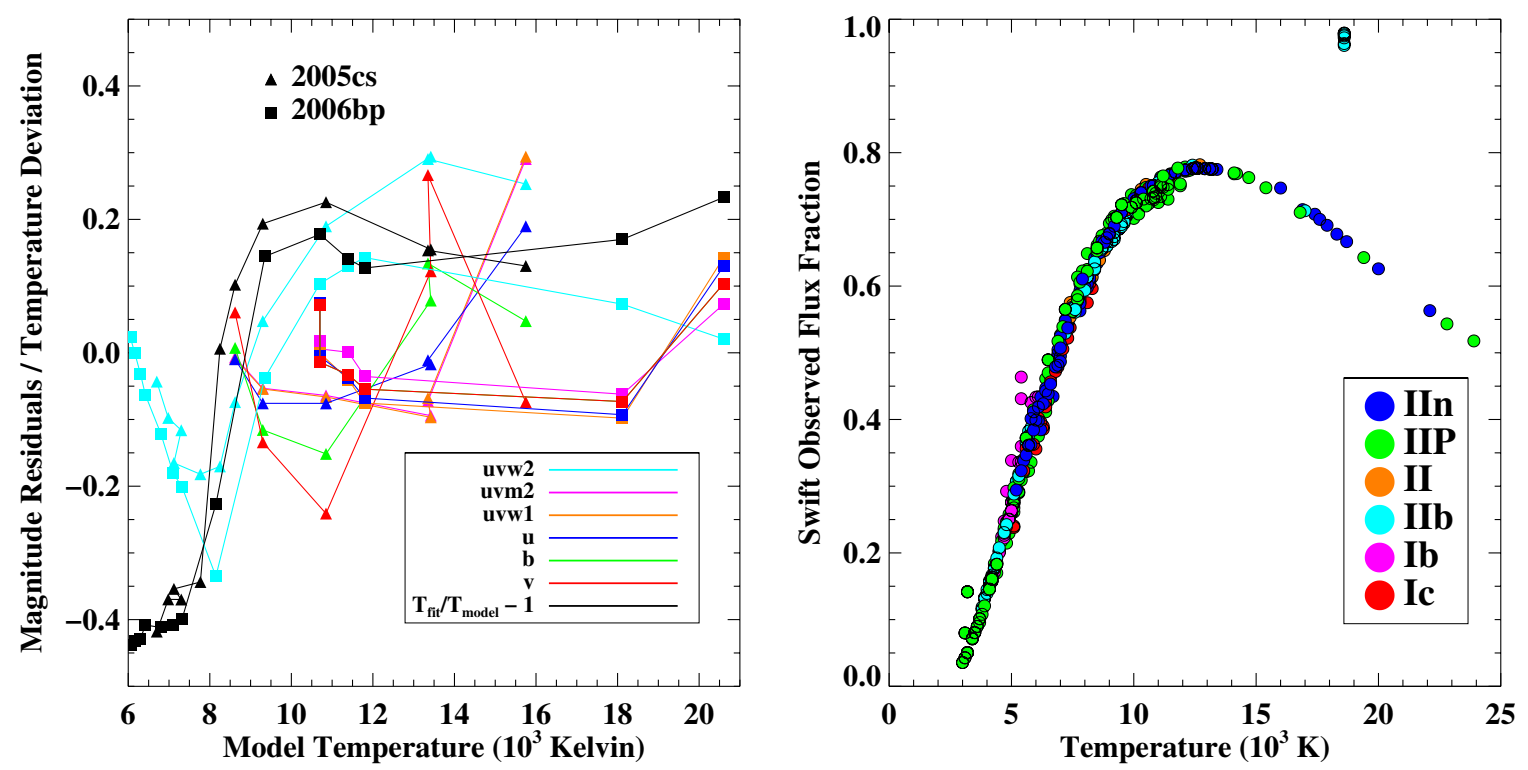

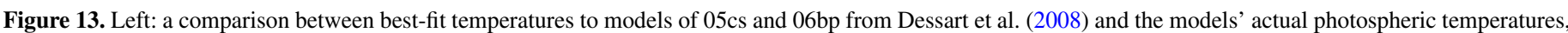

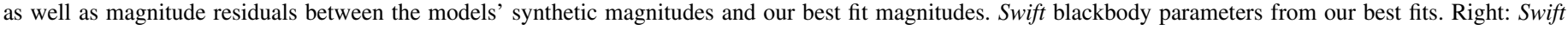

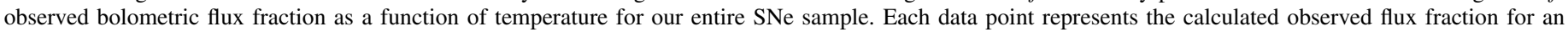
individual observation of a particular SNe, color-coded by observed SNe subtype.

(A color version of this figure is available in the online journal.) 

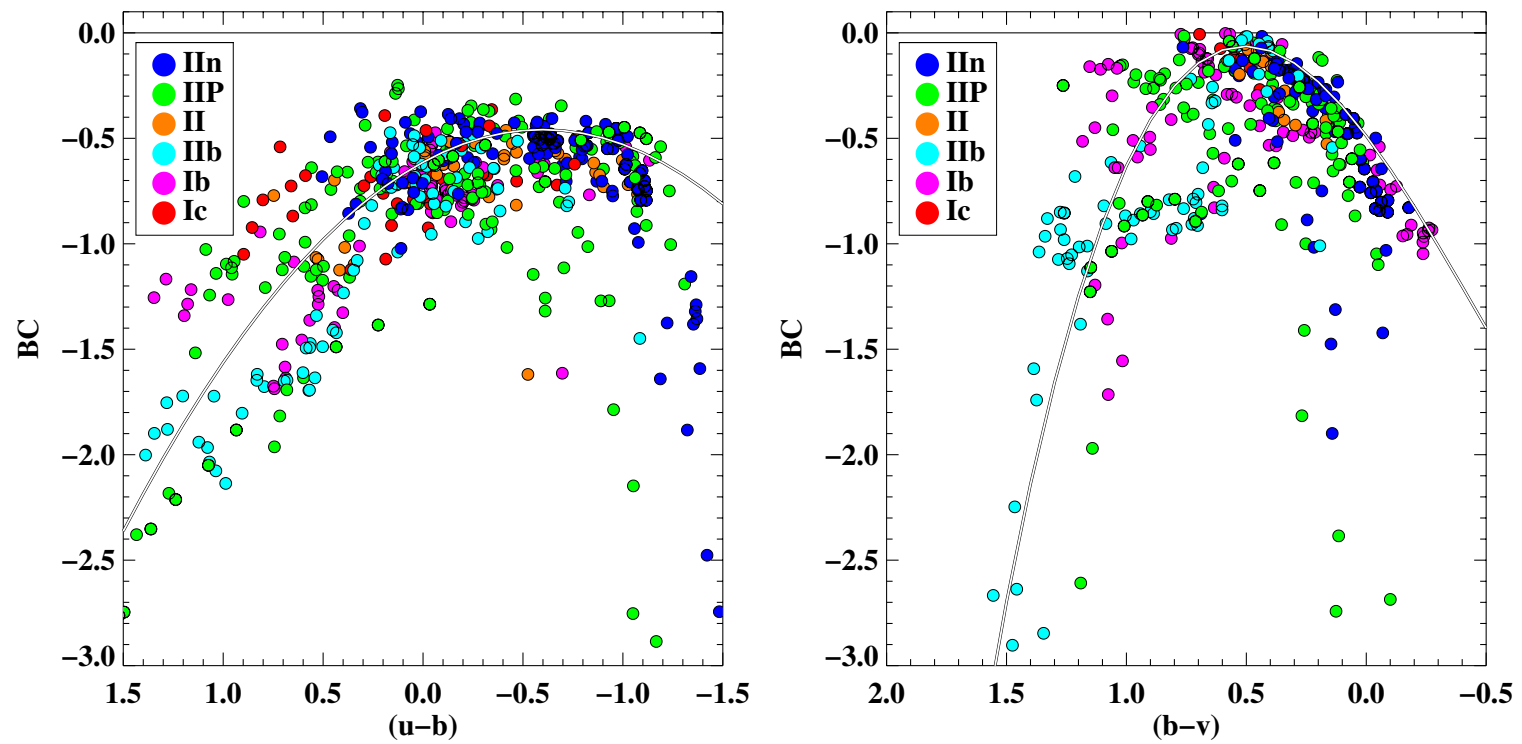

Figure 14. Calculated bolometric correction as a function of optical colors. The $u$ band from Swift has a bluer cutoff than ground based filters. The gray lines represent the Equations (2) (left panel) and (3) (right panel), which are polynomial fits to the observed data discussed in Section 4.2.

(A color version of this figure is available in the online journal.)
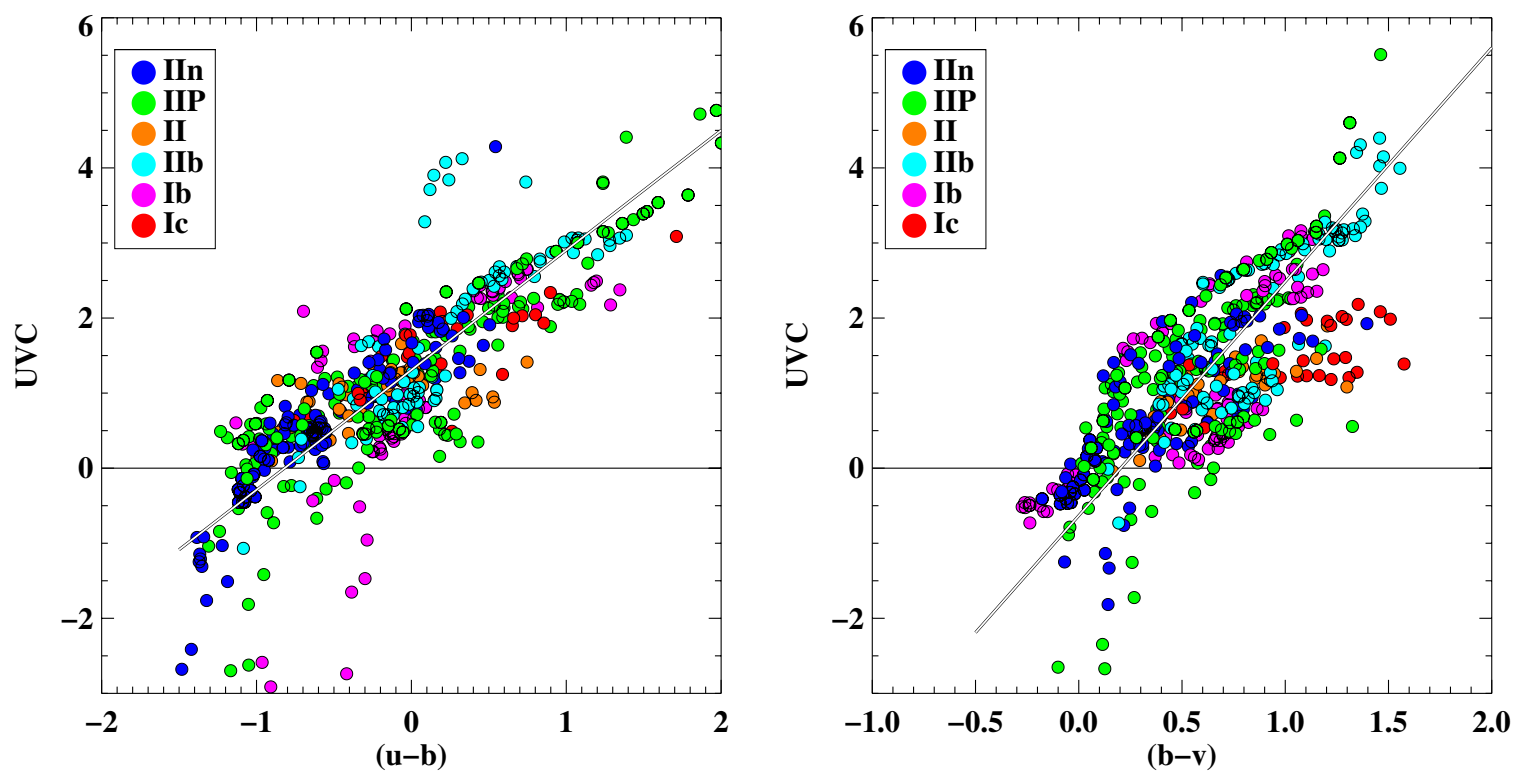

Figure 15. Swift UV filters observed flux + UV flux correction as a function of optical colors. The $u$ band here is from Swift and has a bluer cutoff than ground-based filters. The gray lines represent Equations (4) (left panel) and (5) (right panel), which are linear fits to the observed data discussed in Section 4.2.

(A color version of this figure is available in the online journal.)

the data about these fits are $\sigma=0.34$ and 0.63 for $u-b$ and $b-v$, respectively:

$$
\begin{aligned}
\mathrm{UVC} & =1.268+1.529 \times(u-b), \\
\mathrm{UVC} & =-0.598+3.132 \times(b-v) .
\end{aligned}
$$

\subsection{UV Effect on Bolometric Light Curves}

As we have demonstrated in Sections 4.1 and 4.2, for the $\mathrm{CCSNe}$ with a substantial hydrogen envelope, a substantial amount of an SN's bolometric luminosity lies in the UV regimes at times $\lesssim 50$ days. To illustrate how this may effect the bolometric light curve, we present two Type IIP CCSNe models with slightly varying initial parameters in Figure 16. Both models started with a $23 M_{\odot}$ star evolved until core collapse and then exploded with $5 \times 10^{50}$ erg explosion energy (Young et al. 2006). A wind profile was added to each model with a $10^{8}$ $\mathrm{cm} \mathrm{s}^{-1}$ velocity. Model A has a dense wind created with a mass loss rate of $10^{-5} M_{\odot}$ per year, and Model B has a mass loss rate of $10^{-6} M_{\odot}$ per year. Starting immediately after the launch of the shock wave from core collapse, each SN was evolved with the radiation-hydrodynamics code RAGE (Gittings et al. 2008) and then post-processed with the SPECTRUM code, which uses detailed monochromatic opacities to calculate spectra and light curves (Frey et al. 2013). These models demonstrate that the UV and early time bolometric light curves are very sensitive to the initial progenitor profile and are a valuable addition to constrain models. At these early times, the optical and light curves are similar, but mostly fainter than the UV, and where the UV is dominant we see that small variances in these light curves are 


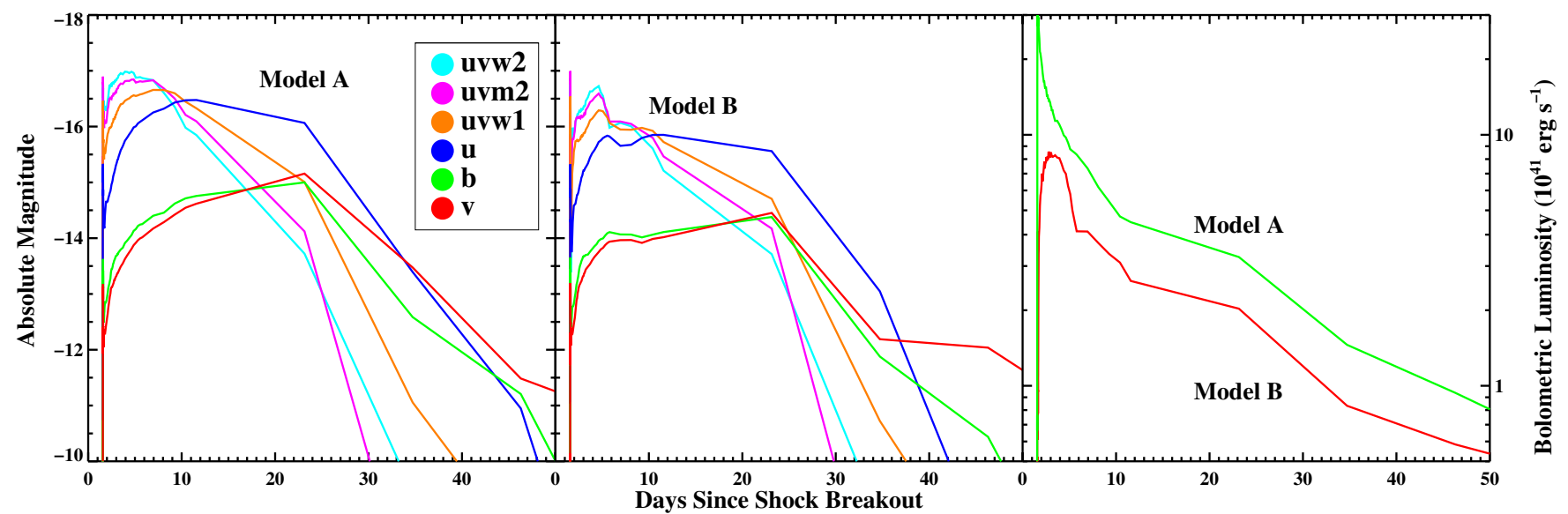

Figure 16. Comparison of two model light curves produced from (Frey et al. 2013). These are model runs from a $23 M_{\odot}$ star, $5 * 10^{50}$ erg explosion energy, and mass loss rates of $10^{-5} / 10^{-6} M_{\odot}$ per year for Models A and B, respectively.

(A color version of this figure is available in the online journal.)

reflected by significant changes in the bolometric light curve. We see this in Figure 16 (right) where the bolometric light curve for model $\mathrm{A}$ has a much brighter, narrower peak than model B, which evidences a more gradual peak followed by a sharp decline. This suggests that to both accurately model the bolometric light curve and the underlying progenitor properties we must be able to incorporate this data.

\subsection{Light Curve and Blackbody Behavior}

When we examine the properties of the best-fit blackbodies we see a number of characteristics that are shared across our observed SNe. For the UV-bright SNe we see that Swift's observed peak UV brightness' have temperatures at above $1.5-2 \times 10^{4}$ Kelvin, and because we often miss the true peak UV brightness (Section 3.4), which happens very rapidly, this may serve as a lower bound for the maximum temperature in the IIP and IIn SNe where this is the case. After Swift's initial observations, we find that this temperature tends to drop rapidly, due to the cooling of the initial shock breakout. In the rare case where we catch this tail in the Ib/c/IIb sample (2010jr, 08ax), we see this same behavior, just on a shorter timescale due to the lack of a thick hydrogen envelope. As our Type IIPs enter the plateau phase we see the best-fit temperatures cluster around $4500-6000 \mathrm{~K}$ values. As Type IIns tend to be very UV bright, we find that they also tend to fit for higher temperatures and have more variability in their cooling curves, with occasional rebrightening evidenced that is constant with CSM interaction reheating the ejecta. In several cases Type IIns have been observed to be very UV bright at months and even years after explosion (Smith et al. 2009; Stritzinger et al. 2012). This general behavior pattern, at early times, is consistent with the late time picture of the SN shockwave interacting with a dense progenitor wind or mass-loss/shell ejection events.

\section{CONCLUSION}

The UV properties of CCSNe are diverse and depend heavily upon the subtype. However, typically the UV's contribution is most important early on, in the $\lesssim 100$ days after shock breakout out when the photosphere is still quite hot. In the rare cases where the UV bands contribute significant flux at late times, it tends to be in IIn SNe where CSM interaction shock heats and excites the gas. The behavior by subtype does appear to be more homogeneous. The Type IIn SNe in our sample are the most varied by subtype-they tend to be UV bright, but their behavior varies significantly in other ways, such as the duration they are able to be observed and their decay rates. On the other hand, Type IIP as a class is the most homogenous, and is well characterized by a linear decline until $\sim 10-20$ days after the $v$-band maximum, at which point the UV light curves settle into a plateau several magnitudes below the optical (cf. Bayless et al. 2013). The $\mathrm{IIb} / \mathrm{Ib} / \mathrm{c}$ SCCSNe fall somewhere in between in terms of homogeneity - they have more individual variation than the IIP - but are more cohesive as a group, even considering that we grouped all three subtypes together for our purposes. As a class they are all UV-faint with UV light curves that have a similar shape to the optical, but are several magnitudes fainter and have a slightly flatter shape. In several rare cases (2008ax, 2010jr) evidence of a shock breakout cooling tail is evident, and it is in these cases only where we tend to see UV-bright behavior. These observations raise a number of questions at the moment for which early time observations are crucial, such as how the pre-explosion progenitor radius and density profile affect early light curve behavior, and the effects of changing local metallicity and extinction upon the observed UV behavior. The advent of extremely high cadence $\mathrm{SNe}$ surveys now coming online and the near-future efforts to observe SNe at rest-frame UV wavelengths at high-redshift, in concert with detailed numerical modeling efforts, offer the promise of future insight into these difficult questions.

When computing bolometric light curves from this sample, we find that Swift's observations do a very good job in the first $\sim 50-100$ days in most cases, albeit with a number of caveats. In the case of SCCSNe, the SNe's lack of a hydrogen envelope means that the blackbody approximation breaks down much more rapidly than for Hydrogen-rich $\mathrm{SNe}$ at the same time as the lack of UV flux makes it harder for Swift to both measure and fit the SNe light curve. For other CCSNe at late times, when the UV flux is faint, additional observations redward of the UVOT band passes are required in order to better constrain the SED shape. Nevertheless, at these early times for the IIP and IIn subtypes we find that up to $\sim 75 \%-80 \%$ of the bolometric flux is in the UV at the brightest of observations, and Swift can reduce this UV extrapolation by a factor of three or more compared to ground-based observations (cf. Bersten \& Hamuy 2009). Using these objects we calculate empirical, bolometric, and UV corrections for use in bolometric light curves calculated from ground-based data. 
We gratefully acknowledge the contributions from members of the Swift UVOT team at the Pennsylvania State University (PSU), University College London/Mullard Space Science Laboratory (MSSL), and NASA/Goddard Space Flight Center. This work is sponsored at PSU and Southwest Research Institute by the NASA ADP grant NNX12AE21G. This research has made use of the NASA/IPAC Extragalactic Database (NED), which is operated by the Jet Propulsion Laboratory, California Institute of Technology, under contract with the National Aeronautics and Space Administration. Report Number LA-UR-13-21329.

\section{REFERENCES}

Andrews, J. E., Gallagher, J. S., Clayton, G. C., et al. 2010, ApJ, 715, 541 Arcavi, I., Gal-Yam, A., Yaron, O., et al. 2011, ApJL, 742, L18 Bayless, A. J., Pritchard, T. A., Roming, P. W. A., et al. 2013, ApJL, 764, L13 Benetti, S., Bufano, F., Vinko, J., et al. 2010, CBET, 2536, 1

Bersten, M. C., \& Hamuy, M. 2009, ApJ, 701, 200

Bloom, J. S., Starr, D. L., Blake, C. H., Skrutskie, M. F., \& Falco, E. E. 2006, in ASP Conf. Ser. 351, Astronomical Data Analysis Software and Systems XV, ed. C. Gabriel, C. Arviset, D. Ponz, \& S. Enrique (San Francisco, CA: ASP), 751

Bock, G., Parker, S., \& Brimacombe, J. 2011, CBET, 2667, 1

Boles, T., Pastorello, A., Stanishev, V., et al. 2011, CBET, 2851, 1

Breeveld, A. A., Curran, P. A., Hoversten, E. A., et al. 2010, MNRAS, 406, 1687

Breeveld, A. A., Landsman, W., Holland, S. T., et al. 2011, in AIP Conf. Proc. 1358, American Institute of Physics Conference Series, ed. J. E. McEnery, J. L. Racusin, \& N. Gehrels (Melville, NY: AIP), 373

Brown, P. J., Holland, S. T., Immler, S., et al. 2009, AJ, 137, 4517

Brown, P. J., Roming, P. W. A., Milne, P., et al. 2010, ApJ, 721, 1608

Campana, S., Mangano, V., Blustin, A. J., et al. 2006, Natur, 442, 1008

Cao, Y., Kasliwal, M. M., Wallerstein, G., Ritchey, A., \& Howell, D. A. 2012, CBET, 2975, 1

Cardelli, J. A., Clayton, G. C., \& Mathis, J. S. 1989, ApJ, 345, 245

Challis, P., Kirshner, R., \& Smith, N. 2010, CBET, 2548, 1

Chatzopoulos, E., Wheeler, J. C., Vinko, J., et al. 2011, ApJ, 729, 143

Chevalier, R. A., \& Soker, N. 1989, ApJ, 341, 867

Chu, J., Li, W., Filippenko, A. V., Blondin, S., \& Narayan, G. 2008, CBET, 1271,1

Ciabattari, F., \& Mazzoni, E. 2011, CBET, 2887, 1

Colesanti, C., Pimentel, E., \& Napoleao, T. 2008, CBET, 1214, 1

Cooke, J., Ellis, R. S., Sullivan, M., et al. 2011, ApJL, 727, L35

Corsi, A., Ofek, E. O., Frail, D. A., et al. 2011, ApJ, 741, 76

Cortini, G., \& Dimai, A. 2009, CBET, 1764, 1

Dessart, L., Blondin, S., Brown, P. J., et al. 2008, ApJ, 675, 644

Dessart, L., \& Hillier, D. J. 2005, A\&A, 437, 667

Dessart, L., \& Hillier, D. J. 2010, MNRAS, 405, 2141

Dintinjana, B., Mikuz, H., Trondal, O., et al. 2006, IAUC, 8687, 1

Drake, A. J., Howerton, S., McNaught, R., et al. 2012, ATel, 4334, 1

Elias-Rosa, N., van Dyk, S. D., Agnoletto, I., \& Benetti, S. 2009, CBET, 1765,1

Elias-Rosa, N., Van Dyk, S. D., Li, W., et al. 2010, ApJL, 714, L254

Filippenko, A. V. 1997, ARA\&A, 35, 309

Filippenko, A. V. 2005, NuPhA, 758, 3

Filippenko, A. V., Li, W. D., Treffers, R. R., \& Modjaz, M. 2001, in ASP Conf. Ser. 246, IAU Colloq. 183: Small Telescope Astronomy on Global Scales, ed. B. Paczynski, W.-P. Chen, \& C. Lemme (San Francisco, CA: ASP), 121

Foley, R. J., Filippenko, A. V., Aguilera, C., et al. 2008, ApJ, 684, 68

Frey, L. H., Even, W., Whalen, D. J., et al. 2013, ApJS, 204, 16 Gal-Yam, A. 2012, Sci, 337, 927

Gal-Yam, A., Cenko, S. B., Fox, D. B., et al. 2007, in AIP Conf. Proc. 924, The Multicolored Landscape of Compact Objects and Their Explosive Origins, ed. T. di Salvo, G. L. Israel, L. Piersant et al. (Melville, NY: AIP), 297

Gehrels, N., Chincarini, G., Giommi, P., et al. 2004, ApJ, 611, 1005

Gezari, S., Halpern, J. P., Grupe, D., et al. 2009, ApJ, 690, 1313

Gittings, M., Weaver, R., Clover, M., et al. 2008, CS\&D, 1, 015005

Green, D. W. E. 2010, CBET, 2397, 1

Hamuy, M., Folatelli, G., Morrell, N. I., et al. 2006, PASP, 118, 2

Hamuy, M., \& Pinto, P. A. 2002, ApJL, 566, L63

Hoeflich, P., Wheeler, J. C., Hines, D. C., \& Trammell, S. R. 1996, ApJ, 459, 307

Humphreys, R. M., Davidson, K., Jones, T. J., et al. 2012, arXiv e-prints

Hurst, G. M., \& Birtwhistle, P. 2005, IAUC, 8555, 3
Inserra, C., Pastorello, A., Turatto, M., et al. 2013, A\&A, 555, A142

Inserra, C., Turatto, M., Pastorello, A., et al. 2011, MNRAS, 417, 261

Itagaki, K., Nakano, S., Puckett, T., et al. 2006, IAUC, 8762, 1

Itagaki, K., Nakano, S., Quimby, R., et al. 2007, IAUC, 8843, 1

Joubert, N., Li, W., Christensen, E. J., et al. 2007, IAUC, 8813, 1

Kasen, D., \& Woosley, S. E. 2009, ApJ, 703, 2205

Krisciunas, K., Suntzeff, N. B., Phillips, M. M., et al. 2004, AJ, 128, 3034

Law, N. M., Kulkarni, S. R., Dekany, R. G., et al. 2009, PASP, 121, 1395

Leonard, D. C., Filippenko, A. V., Li, W., et al. 2002, AJ, 124, 2490

Levesque, E. M., Stringfellow, G. S., Ginsburg, A. G., Bally, J., \& Keeney, B. A. 2013, arXiv:1211.4577

Li, G., Cenko, S. B., Li, W., et al. 2011, CBET, 2721, 1

Li, W., Cenko, S. B., \& Filippenko, A. V. 2009, CBET, 1952, 1

Li, W., Jha, S., Filippenko, A. V., et al. 2006, PASP, 118, 37

Luppi, F., Yusa, T., Koff, R. A., et al. 2012, CBET, 2974, 2

Maguire, K., Jerkstrand, A., Smartt, S. J., et al. 2012a, MNRAS, 420, 3451

Maguire, K., Sullivan, M., Ellis, R. S., et al. 2012b, MNRAS, 426, 2359

Matheson, T., Kirshner, R. P., Challis, P., et al. 2008, AJ, 135, 1598

Mauerhan, J. C., Smith, N., Filippenko, A., et al. 2013a, MNRAS, 430, 1801

Mauerhan, J. C., Smith, N., Silverman, J. M., et al. 2013b, MNRAS, 431, 2599

Maza, J., Hamuy, M., Antezana, R., et al. 2010a, CBET, 2125, 1

Maza, J., Hamuy, M., Antezana, R., et al. 2010b, CBET, 2544, 1

Mikuz, H., \& Maticic, S. 2007, CBET, 1116, 1

Miller, A. A., Chornock, R., Perley, D. A., et al. 2009, ApJ, 690, 1303

Modjaz, M., Li, W., Butler, N., et al. 2009, ApJ, 702, 226

Monard, L. A. G. 2009, CBET, 2071, 1

Monard, L. A. G. 2010, CBET, 2250, 1

Monard, L. A. G., Martin, R., Ponticello, N. J., et al. 2006, IAUC, 8693, 1

Monard, L. A. G., Puckett, T., \& Gray, P. 2007, IAUC, 8814, 2

Mostardi, R., Li, W., \& Filippenko, A. V. 2008, CBET, 1280, 1

Nakano, S. 2010, CBET, 2281, 1

Nakano, S., \& Itagaki, K. 2006, IAUC, 8700, 4

Nakano, S., Kadota, K., \& Buzzi, L. 2009a, CBET, 1670, 1

Nakano, S., Kadota, K., Ikari, Y., \& Itagaki, K. 2009b, CBET, 1718, 1

Nakano, S., Kadota, K., Itagaki, K., \& Corelli, P. 2008a, IAUC, 8908, 2

Nakano, S., Kadota, K., Kryachko, T., \& Korotkiy, S. 2008b, CBET, 1626, 1

Nakano, S., Kadota, K., \& Wells, W. 2008c, CBET, 1636, 1

Nakano, S., Yusa, T., \& Kadota, K. 2009c, CBET, 2006, 1

Newton, J., \& Puckett, T. 2010, CBET, 2532, 1

Nissinen, M., \& Oksanen, A. 2008, CBET, 1324, 1

Oates, S. R., Bayless, A. J., Stritzinger, M. D., et al. 2012, MNRAS, 424, 1297

Ofek, E. O., Howell, D. A., Kasliwal, M. M., et al. 2010, CBET, 2198, 1

Otsuka, M., Meixner, M., Panagia, N., et al. 2012, ApJ, 744, 26

Panagia, N. 2003, in Supernovae and Gamma-Ray Bursters, ed. K. Weiler (Lecture Notes in Physics, Vol. 598, Berlin: Springer), 113

Parisky, X., \& Li, W. 2007, CBET, 1129, 1

Pastorello, A., Cappellaro, E., Inserra, C., et al. 2013, ApJ, 767, 1

Pastorello, A., Kasliwal, M. M., Crockett, R. M., et al. 2008, MNRAS, 389, 955

Pastorello, A., Smartt, S. J., Mattila, S., et al. 2007, Natur, 447, 829

Pignata, G., Cifuentes, M., Maza, J., et al. 2010a, CBET, 2269, 1

Pignata, G., Cifuentes, M., Maza, J., et al. 2010b, CBET, 2545, 1

Poole, T. S., Breeveld, A. A., Page, M. J., et al. 2008, MNRAS, 383, 627

Prieto, J. L., Brimacombe, J., Drake, A. J., \& Howerton, S. 2013, ApJL, 763, L27

Pritchard, T. A., Roming, P. W. A., Brown, P. J., et al. 2012, ApJ, 750, 128

Puckett, T., \& Pelloni, A. 2005, CBET, 285, 1

Quadri, U., Strabla, L., Girelli, R., et al. 2012, CBET, 3054, 1

Rau, A., Kulkarni, S. R., Law, N. M., et al. 2009, PASP, 121, 1334

Rich, D. 2010, CBET, 2207, 1

Roming, P. W. A., Kennedy, T. E., Mason, K. O., et al. 2005, SSRv, 120, 95

Roming, P. W. A., Pritchard, T. A., Brown, P. J., et al. 2009, ApJL, 704, L118

Roming, P. W. A., Pritchard, T. A., Prieto, J. L., et al. 2012, ApJ, 751, 92

Rostopchin, S., \& Riley, V. 2008, CBET, 1262, 2

Roy, R., Kumar, B., Benetti, S., et al. 2011, ApJ, 736, 76

Schlafly, E. F., \& Finkbeiner, D. P. 2011, ApJ, 737, 103

Silverman, J. M., Filippenko, A. V., \& Cenko, S. B. 2011, ATel, 3398, 1

Smartt, S. J. 2009, ARA\&A, 47, 63

Smith, N., Cenko, S. B., Butler, N., et al. 2012a, MNRAS, 420, 1135

Smith, N., \& Mauerhan, J. 2012, ATel, 4412, 1

Smith, N., Mauerhan, J. C., Silverman, J. M., et al. 2012b, MNRAS, 426, 1905

Smith, N., Silverman, J. M., Chornock, R., et al. 2009, ApJ, 695, 1334

Smith, N., Silverman, J. M., Filippenko, A. V., et al. 2012c, AJ, 143, 17

Soderberg, A. M., Margutti, R., Zauderer, B. A., et al. 2012, ApJ, 752, 78

Sparre, M., Sollerman, J., Fynbo, J. P. U., et al. 2011, ApJL, 735, L24

Starling, R. L. C., Page, K. L., Pe'Er, A., Beardmore, A. P., \& Osborne, J. P. 2012, MNRAS, 427, 2950

Stoll, R., Prieto, J. L., Stanek, K. Z., et al. 2011, ApJ, 730, 34 
Stritzinger, M., Mazzali, P., Phillips, M. M., et al. 2009, ApJ, 696, 713 Stritzinger, M., Morrell, N., \& Phillips, M. M. 2010, CBET, 2220, 1 Stritzinger, M., Taddia, F., Fransson, C., et al. 2012, ApJ, 756, 173

Tomasella, L., Benetti, S., Pastorello, A., \& Valenti, S. 2012, CBET, 3032, 2

Tomasella, L., Benetti, S., Pastorello, A., et al. 2011, CBET, 2941, 2

Tominaga, N., Tanaka, M., Nomoto, K., et al. 2005, ApJL, 633, L97

Turatto, M., Benetti, S., Cappellaro, E., \& Bufano, F. 2010, CBET, 2252, 1
Valenti, S., Pastorello, A., Benetti, S., et al. 2011, CBET, 2906, 2 Valenti, S., Taubenberger, S., Pastorello, A., et al. 2012, ApJL, 749, L28 Vinko, J., Zheng, W., Romadan, A., et al. 2010, CBET, 2556, 1 Winslow, D., Li, W., \& Filippenko, A. V. 2008, CBET, 1279, 1 Wood-Vasey, W. M., Miknaitis, G., Stubbs, C. W., et al. 2007, ApJ, 666, 694 Young, P. A., Fryer, C. L., Hungerford, A., et al. 2006, ApJ, 640, 891 Yuan, F., Quimby, R., McKay, T., et al. 2008, CBET, 1462, 1 